\title{
Pig genome functional annotation enhances biological interpretations of complex traits and comparative epigenomics
}

Huaijun Zhou ( $\nabla$ hzhou@ucdavis.edu )

University of California, Davis https://orcid.org/0000-0001-6023-9521

\section{Zhangyuan Pan}

Department of Animal Science, University of California, Davis

\section{Yuelin Yao}

The University of Edinburgh

\section{Hongwei Ying}

Agricultural Genome Institute at Shenzhen, Chinese Academy of Agricultural Sciences

\section{Zexi Cai}

Center for Quantitative Genetics and Genomics, Faculty of Technical Sciences, Aarhus University https://orcid.org/0000-0002-9579-3415

\section{Ying Wang}

UC Davis

\section{Lijing Bai}

Agricultural Genome Institute at Shenzhen, Chinese Academy of Agricultural Sciences

\section{Colin Kern}

University of California, Davis https://orcid.org/0000-0002-3249-6405

\section{Michelle Halstead}

University of California, Davis https://orcid.org/0000-0003-0168-2704

\section{Kelly Chanthavixay}

Department of Animal Science, University of California, Davis,

\section{Nares Trakooljul}

https://orcid.org/0000-0002-3214-2498

\section{Klaus Wimmers}

Leibniz-Institute for Farm Animal Biology (FBN) https://orcid.org/0000-0002-9523-6790

\section{Goutam Sahana}

Center for Quantitative Genetics and Genomics, Faculty of Technical Sciences

\section{Guosheng Su}

Center for Quantitative Genetics and Genomics, Faculty of Technical Sciences

\section{Mogens Sandø Lund}

Center for Quantitative Genetics and Genomics, Faculty of Technical Sciences 


\section{Merete Fredholm}

University of Copenhagen

\section{Peter Karlskov-Mortensen}

University of Copenhagen https://orcid.org/0000-0002-2491-2763

\section{Catherine Ernst}

Agricultural Research Service https://orcid.org/0000-0003-2833-0995

\section{Pablo Ross}

University of California, Davis https://orcid.org/0000-0002-3972-3754

\section{Christopher Tuggle}

lowa State University https://orcid.org/0000-0002-4229-5316

\section{Lingzhao Fang}

IGMM

\section{Article}

Keywords: livestock genome, epigenetics

Posted Date: March 22nd, 2021

DOI: https://doi.org/10.21203/rs.3.rs-253276/v1

License: (c) (1) This work is licensed under a Creative Commons Attribution 4.0 International License. Read Full License

Version of Record: A version of this preprint was published at Nature Communications on October 6th, 2021. See the published version at https://doi.org/10.1038/s41467-021-26153-7. 
1 Pig genome functional annotation enhances biological interpretations of complex traits and

2 comparative epigenomics

5 Zhangyuan Pan ${ }^{1 \#}$, Yuelin Yao ${ }^{2 \#}$, Hongwei Yin ${ }^{3}$, Zexi Cai ${ }^{4}$, Ying Wang ${ }^{1}$, Lijing Bai ${ }^{3}$, Colin Kern ${ }^{1}$,

6 Michelle Halstead $^{1}$, Kelly Chanthavixay ${ }^{1}$, Nares Trakooljul $^{5}$, Klaus Wimmers ${ }^{5}$, Goutam Sahana ${ }^{4}$,

7 Guosheng Su${ }^{4}$, Mogens Sandø Lund ${ }^{4}$, Merete Fredholm ${ }^{6}$, Peter Karlskov-Mortensen ${ }^{6}$, Catherine

8 W. Ernst ${ }^{7}$, Pablo Ross ${ }^{1}$, Christopher K. Tuggle ${ }^{8}$, Lingzhao Fang ${ }^{2 *}$, Huaijun Zhou ${ }^{1 *}$

$10{ }^{1}$ Department of Animal Science, University of California, Davis, Davis, CA, USA

$11{ }^{2}$ MRC Human Genetics Unit at the Institute of Genetics and Molecular Medicine, The University

12 of Edinburgh, Edinburgh EH4 2XU, UK

$13{ }^{3}$ Agricultural Genome Institute at Shenzhen, Chinese Academy of Agricultural Sciences, Shenzhen $14 \quad 518120$, China

$15{ }^{4}$ Center for Quantitative Genetics and Genomics, Faculty of Technical Sciences, Aarhus

16 University, Tjele 8300, Denmark

$17 \quad{ }^{5}$ Leibniz-Institute for Farm Animal Biology, Dummerstorf, Germany

$18{ }^{6}$ Animal Genetics, Bioinformatics and Breeding, Department of Veterinary and Animal Sciences,

19 University of Copenhagen, Frederikgsberg C 1870, Denmark

$20 \quad{ }^{7}$ Department of Animal Science, Michigan State University East Lansing, MI, USA

$21{ }^{8}$ Department of Animal Science, Iowa State University, Ames, IA, USA 
24 "These authors contributed equally

$25 *$ Corresponding authors

26 HZ: Department of Animal Science, University of California, Davis, CA, 95616, USA

27 E-mail: hzhou@ucdavis.edu

28 LF: MRC Human Genetics Unit at the Institute of Genetics and Molecular Medicine, The

29 University of Edinburgh, Edinburgh EH4 2XU, UK

$30 \quad$ E-mail: Lingzhao.fang@igmm.ed.ac.uk

31

32

33

34

35

36

37

38

39

40

41

42

43

44

45 


\section{Abstract}

47 The functional annotation of livestock genomes is crucial for understanding the molecular

48 mechanisms that underpin complex traits of economic importance, adaptive evolution and

49 comparative genomics. Here, we provide the most comprehensive catalogue to date of regulatory

50 elements in the pig (Sus scrofa) by integrating 223 epigenomic and transcriptomic data sets,

51 representing 14 biologically important tissues. We systematically describe the dynamic epigenetic

52 landscape across tissues by functionally annotating 15 different chromatin states and defining their

53 tissue-specific regulatory activities. We demonstrate that genomic variants associated with

54 complex traits and adaptive evolution in pig are significantly enriched in active promoters and

55 enhancers. Furthermore, we reveal distinct tissue-specific regulatory selection between Asian and

56 European pig domestication processes. Compared with human and mouse epigenomes, we show

57 that porcine regulatory elements are more conserved in DNA sequence, under both rapid and slow

58 evolution, than those under neutral evolution across pig, mouse, and human. Finally, we provide

59 novel biological insights on tissue-specific regulatory conservation and demonstrate that,

60 depending on the traits, mouse or pig might be more appropriate biomedical models for different

61 complex traits and diseases in humans through integrating comparative epigenomes with 47 human

62 genome-wide association studies. 


\section{Main}

65 Functional elements play essential roles in regulating gene expression in living cells and tissues ${ }^{1}$.

66 There have been great efforts on the identification and annotation of functional elements in human

67 and mouse genomes ${ }^{1-11}$ as well as other model organisms including Drosophila ${ }^{12}$ and C. elegans ${ }^{13}$.

68 Significant enrichment of variants associated with human complex traits within regulatory

69 elements has demonstrated the importance of Encyclopedia of DNA Elements (ENCODE) data ${ }^{14}$.

70 Comparative analysis of epigenomes and transcriptomes across species could provide novel

71 insights into the interrogation of underlying molecular mechanisms of human disease ${ }^{8,15}$. Genetic

72 variants associated with common illnesses are enriched in human orthologues of mouse regulatory

73 elements identified by ENCODE3 ${ }^{9}$, which validates the mouse could serve as a biomedical model

74 for understanding some human diseases. However, compared with the mouse, pig (Sus scrofa) has

75 more anatomical and physiological similarities to humans ${ }^{16-18}$, and has been widely used as a

76 human medical model ${ }^{16,17,19-21}$. The pig is also one of the most important farm animal species for

77 meat production worldwide ${ }^{22}$. The genetic improvement of economically important complex traits

78 such as growth, feed efficiency, and health contribute to efficient and sustainable production of

79 animal protein ensuring a secure food supply for a growing world population. Functional

80 annotation of regulatory elements in pig will lay a solid foundation for the identification of

81 causative variants associated with phenotypic variations of complex traits, due to the enrichment

82 of these variants in regulatory regions and the small genetic effect of each variant, making them

83 difficult to discover ${ }^{23}$.

84 Following ENCODE and Roadmap Epigenomics projects ${ }^{8}$, the Functional Annotation of 85 Animal Genomes (FAANG) initiative ${ }^{24}$, although still in its infancy, has made great progress 
86 towards annotating functional elements in many tissues across multiple domestic species including

87 pigs $^{25-30}$. Here, we present 95 new genome-wide sequencing datasets from six gut-associated

88 porcine tissues and integrate them with 128 previously published FAANG datasets from eight

89 biologically distinct tissues. The collective interpretation of these datasets yields the most

90 comprehensive annotation of functional elements to date in any domesticated animal species. In

91 addition, we find that tissue-specific regulatory elements were enriched for the potential causative

92 variants of complex phenotypes by integrating a large scale of genome-wide association studies

93 (GWAS) and expression QTL (eQTL) datasets. Furthermore, by combining selection signature of

94 the pig genome, we show that tissue-specific regulatory elements play an important role during

95 the domestication. Finally, we compared porcine functional annotations with complementary

96 datasets from the human and mouse and integrated GWAS datasets concerning 47 human complex

97 traits. These comparisons demonstrate conservation of tissue-specific epigenetic signatures,

98 suggesting that, depends on diseases, pig or mouse may be a better animal model for them than

99 the other one.

100

\section{Results}

\section{Data summary}

103 We integrated 223 genome-wide sequencing datasets from 14 major tissues in pig (Fig. 1a), 104 representing four histone modifications (H3K4me3, H3K4me1, H3K27ac and H3K27me3) 105 measured by Chromatin Immunoprecipitation sequencing (ChIP-seq), chromatin accessibility by 106 the Assay for Transposase-Accessible Chromatin (ATAC-seq), DNA methylation by Reduced 107 Representation Bisulfite sequencing (RRBS), and gene expression by RNA-seq (Supplementary 108 Fig. 1). We produced nearly 9 billion mapped reads with an average remaining rate of $68.81 \%$ 
across samples (Supplementary Table 1). Among 14 tissues, we obtained an average of 32,387,

$110106,849,72,252,98,721$, and 122,585 peaks for H3K4me3, H3K4me1, H3K27ac, H3K27me3,

111 and ATAC, covering 1.56, 2.78, 2.37, 7.74, and 3.31\% of the entire genome, respectively (Fig. 1b,

112 c). Additionally, we utilized 16 CTCF ChIP-seq datasets from eight tissues ${ }^{29}$ and four Hi-C datasets

113 from liver ${ }^{30}$ to identify topologically associating domains (TADs) and Hi-C loop for associating

114 regulatory elements (enhancers) with potential target genes.

115 The hierarchical clustering of samples based on the signal intensity of epigenetic marks and

116 gene expression profiles clearly recapitulated sequencing assays, followed by tissue types and

117 biological replicates (Fig. 1d), which was consistent with results of principal component analysis

118 (PCA) (Supplementary Fig. 2). The six assays formed three major clusters: (1) active regulatory

119 regions (H3K4me3, H3K27ac, H3K4me1 and ATAC), (2) Polycomb repression (H3K27me3), and

120 (3) gene expression (RNA-seq). The four active regulatory marks were positively correlated with

121 each other, but were negatively correlated with $\mathrm{H} 3 \mathrm{~K} 27 \mathrm{me} 3$, especially H3K27ac. The signal

122 intensity of RNA-seq (within gene bodies) showed a weakly positive correlation with active

123 regulatory marks, and a negative correlation with $\mathrm{H} 3 \mathrm{~K} 27 \mathrm{me} 3$. Overall, all four active regulatory

124 marks exhibited significant enrichments in the upstream of transcription start sites (TSS) of genes

125 across tissues (Fig. 1e).

126 We present Myosin IA (MYO1A) gene, which is engaged in responses to Escherichia coli

127 infection and microvillar membrane morphology in intestinal tissues ${ }^{31,32}$, as an example to show

128 the complex interplays of regulatory elements and gene expression in Figure 1f. MYO1A is

129 specifically and highly expressed in intestine tissues, and showed specific enrichments of

$130 \mathrm{H} 3 \mathrm{~K} 27 \mathrm{ac}$ signals around its TSS in intestine tissues but not in other tissues (Fig. 1f). In addition, 
131 the TSS of MYO1A is accessible and was enriched for other active regulatory marks (i.e., H3K27ac,

132 H3K4me3 and H3K4me1) but not for Polycomb repression (H3K27me3) (Fig. 1f).

\section{Prediction and characterization of chromatin states across 14 tissues}

134 We defined 15 distinct chromatin states by combining all five epigenetic marks across 14 tissues.

135 These states mainly represented promoters (TssA, TssAHet and TssBiv, covering $1.16 \%$ of the

136 entire genome), TSS-proximal transcribed regions (TxFlnk, TxFlnkWk and TxFlnkHet, covering

$1370.92 \%$ of the genome), enhancers (EnhA, EnhAMe, EnhAWk, EnhAHet and EnhPois, covering

$1386.5 \%$ of the genome), repressed regions (Repr and ReprWk, covering $13.25 \%$ of the genome) and

139 quiescent regions (73.39\%) (Fig. 2a-e, Supplementary Table 2). Totally we identified 2,097,958

140 regulatory elements (exclude Qui) spanning 14 tissues including 39.351 active promoters (TssA),

141188,827 active strong enhancers (EnhA), and 142,821 repressors (Repr) (Supplementary Fig. 4a-

142 c). On average, $4.79 \%$ of the genome was accessible but did not coincide any other measured

143 epigenetic marks (ATAC islands), indicating that additional epigenetic marks are required to

144 further explore the biological function of such regions. TssA and TssBiv showed the highest

145 enrichment of conserved DNA sequence elements, followed by TSS-proximal transcribed regions

146 and accessible enhancers (EnhA and EnhAMe) (Fig. 2f). In general, TssA and TssBiv showed the

147 highest enrichment at TSS, while other chromatin states showed enrichment at up- and down-

148 stream of TSS (Fig. 2g). For instance, TssAHet and TSS-proximal transcribed states had the

149 highest enrichment around $2 \mathrm{~kb}$ up-stream of TSS, whereas enhancer states showed the highest

150 enrichment around 20kb up-stream of TSS. Repressed states were enriched around 20kb up- and

151 down-stream of TSS (Fig. 2g).

152 In general, different chromatin states showed distinct DNA methylation levels (Fig. 2h).

153 Promoter and TSS-proximal transcribed states were hypomethylated compared to nearby sequence 
154 (10kb up- and down-stream of TSS). Among promoter states, TssA had the lowest methylation

155 level, confirming the well-known negative correlation between promoter methylation and gene

156 expression $^{33}$. The enhancer states showed intermediate methylation levels, among which EnhA and

157 EnhAMe had lower methylation levels compared to other enhancers (Fig. 2g), where we also

158 observed that EnhA and EnhAMe had more conserved sequence than other enhancers (Fig. 2f).

159 This result suggests accessible enhancers may have more conserved sequences than non-accessible

160 enhancers.

161 We took chromosome $7(\mathrm{Chr} 7)$ as an example to explore the relationships among chromatin

162 states, individual epigenetic marks, gene density, gene expression, DNA methylation and

163 chromatin conformation (Fig. 2i). For instance, we observed that regions with higher density of

164 genes were characterized by active chromatin states, higher gene expression, more chromatin

165 accessibility, and lower methylation level. The chromatins were more physically interacted

166 (measured by TADs from Hi-C data) within both gene desert and gene rich regions than the rest

167 of genomic regions. To examine the associations of chromatin states with gene expression across

168 tissues, we presented the VIL1 locus (Villin-1), which participates in response to

169 intestinal inflammation ${ }^{34}$, as an example (Fig. 2j). VIL1 exhibited tissue-specific active promoters

170 and enhancers, as well as high expression in intestinal tissues compared to other tissues. Of

171 particular note, despite the presence of TssA, VIL1 was not expressed in stomach, possible due to

172 the lack of enhancer activity in the upstream of its TSS, indicating that enhancers together with

173 promoters may collectively regulate gene expression. Similar patterns were observed for MYO1A

174 and $H N F 4 G$ (Supplementary Fig. 3).

175 Dynamics of chromatin states across genome and tissues 
We clustered the entire genome into 12 modules based on their relative frequency of chromatin

177 states and observed that these modules exhibited distinct enrichments for protein-coding genes,

178 non-coding genes and CpG islands (Fig. 3a). For instance, module 2 (M2) was characterized by

179 active promoters and accessible enhancers, showed the highest enrichment for genes and CpG

180 islands, and the lowest levels of DNA methylation, and the highest gene expression levels (Fig.

$1813 b)$. Compared to modules 11 and 12, module 10 showed similar enrichment for Polycomb

182 repression but higher enrichment for TssBiv, in which genes exhibited significantly lower

183 expression levels, suggesting the crucial role of TssBiv for regulating gene repression (Fig. 3b). In

184 addition, we noticed that module 1 had high enrichment for TssAHet, high levels of DNA

185 methylation, and high representation of genes located on the $\mathrm{X}$ chromosome and these genes are

186 relevant with histone modification Gene Ontology (GO) terms (Supplementary Table 3). This may

187 indicate potential roles of TssAHet in heterochromatin on the Chromosome $\mathrm{X}^{35}$.

188 By examining the distribution of chromatin states among all 14 tissues, we found that enhancer

189 activity was the most variable between tissues, while promoters were least variable (Fig. 3c-d,

190 Supplementary Fig. 4d,e). Among promoters, TssBiv was least constitutive and often switched to

191 TSS-proximal transcribed or quiescent regions between tissues (Fig. 3d). Hierarchical clustering

192 of samples using the signal intensity of H3K4me1 within EnhA clearly separated different tissue

193 types (Fig. 3e), suggesting that the signal intensity of individual epi-mark in enhancers is highly

194 indicative of tissue identity.

195 To explore the relationship between proximal regulatory elements (within $2 \mathrm{~kb}$ of TSS of genes)

196 and tissue-specific gene expression, we identified genes with tissue-specific expression (TSE),

197 which were significantly engaged in known biological functions of specific tissues (Supplementary

198 Fig. 5, Supplementary Table 4). We also observed that TSE were enriched for active states 
199 (promoters, transcribed regions and enhancers) and depleted for repressed states in the $2 \mathrm{~kb}$ regions 200 around their TSS in the corresponding tissue compared to other tissues (Fig. 3f). Furthermore, we

201 found that predicted target enhancers of TSE in a tissue were more constitutive among biologically 202 similar tissues compared with other tissues (Fig. 3g), which was consistent with promoters of TSE 203 (Supplementary Fig. 6).

\section{Functional characterization of tissue-specific chromatin states}

205 As enhancers were most variable among tissues compared to other chromatin states, we 206 identified an average of 6,895 tissue-specific EnhAs among 14 tissues, ranging from 1,393 in 207 jejunum to 14,811 in skeletal muscle (Fig. 4a). To further investigate the biological functions of 208 such enhancers, we defined three other types of EnhA, including all-common EnhA (shared among 209 all tissues), gut-common EnhA (shared among gut tissues) and brain-common EnhA (shared 210 among brain tissues). Gene Ontology (GO) analysis of putative target genes of these different types 211 of EnhAs revealed distinct biological functions (Fig. 4b, Supplementary Table 5). For instance, 212 all-common EnhAs were involved in fundamental biological processes (e.g., regulation of mRNA

213 catabolic processes and responses to wounding), whereas gut-common EnhAs were significantly

214 involved in intestinal development, digestion and absorption, and immune response. EnhAs that

215 were specifically active in individual gut tissues showed distinct functions, clearly matching the 216 known biological functions of the tissue in question. For example, jejunum-specific EnhAs were

217 involved in biological processes relevant to T cell and lymphocyte function ${ }^{36}$, while colon-specific 218 EnhAs were mainly engaged in stress-activated MAPK cascades ${ }^{37}$ (Fig. 4b). We observed that 219 intestine- and spleen-specific EnhAs shared many immune functions, and brain-specific EnhAs 220 were significantly involved in memory and learning (Fig. 4b). Furthermore, we observed that 221 genes whose topologically associated with tissue-specific EnhAs (Methods) were specifically 
222 highly expressed in the corresponding tissues (Fig. 4c), and that methylation levels of tissue223 specific EnhAs were lower in the corresponding tissues (Supplementary Fig. 7a), indicating that 224 these tissue-specific enhancers and their methylation level were accurately predicted to regulate 225 the expression of associated target genes.

226 To explore potential tissue-specific transcription factors (TF), first we identified motifs that 227 were significantly enriched in tissue-specific EnhAs (Fig. 4d, Supplementary Fig. 8a), such as 228 MEF2A, HNF1B, and HNF4A1 in muscle, liver and intestinal tissues, respectively, which was in 229 line with previous findings in humans ${ }^{8}$. In addition, we found the binding motif of HNF4G, which 230 participates in the renewal of intestinal stem cells in mice ${ }^{38}$ and is specifically active in intestine 231 (Supplementary Fig. 3b), and is enriched in most intestine-specific EnhAs, whereas CDX2, a major 232 regulator of intestine-specific genes involved in cell growth and differentiation and is highly 233 expressed in jejunum over duodenum and ileum ${ }^{39,40}$, and its motif is specifically enriched in 234 jejunum-specific EnhAs. The expression levels of the inferred TFs were higher in the 235 corresponding tissue than in other tissues (Supplementary Fig. 8b,c), indicating that these tissue236 specific enhancers are hotspots for TF activity and play important roles in the tissue-specific 237 regulation of gene expression. We further observed that genes linked to tissue-specific EnhAs were 238 significantly associated with biologically relevant complex diseases in humans and mice (Fig. 4e,

239 Supplementary Fig. 7b, Supplementary Table 6). For example, colon-specific EnhAs were 240 associated with recurrent bacterial infection diseases, and cecum-specific EnhAs were 241 significantly associated with bruising susceptibility diseases.

242 We also explored potential function for tissue-specific promoters (TssA), and found that 243 promoters also showed tissue-specific regulatory (TSR) function, but to a lesser degree than 244 enhancers (Supplementary Fig. 9, Supplementary Table 7). 


\section{6 and complex traits in pigs}

247 To determine whether genomic regions associated with adaptive evolution are significantly 248 enriched in regulatory elements (REs), we first identified selection signatures (the top 5\% of 249 regions measured by Fst) by comparing wild with domesticated pigs in Asian and European 250 populations separately (Supplementary Table 8). We found that genomic regions under selective 251 pressure were most enriched for TssA and TSS-proximal transcribed regions, followed by 252 enhancers, with similar patterns in both Asian and European populations (Fig. 5a, Supplementary 253 Fig. 10a). In examining tissue-specific regulation, our analysis revealed that the all-common TssA 254 were significantly enriched within regions under selective pressure in both populations (Fig. 5b).

255 Interestingly, spleen-specific REs were most enriched in Asian pig domestication, wheras cortex256 specific REs were most enriched in European pig domestication (Fig. 5b). Consequently, tissue257 specific gene regulation may have played an essential role in the adaptive selection processes that 258 resulted in Asian and European pig domestication. This result was also in agreement with the 259 observation that Asian domesticated pigs being more disease resistant ${ }^{41}$, whereas European 260 domesticated pigs are more active and aggressive ${ }^{42,43}$.

261 To ask whether SNPs associated with complex traits in pigs are enriched in regulatory regions, 262 we integrated GWAS signal enrichment analysis for 44 complex traits (Supplementary Table 9) 263 with all 15 chromatin states, and demonstrated that GWAS signals were most enriched in TssA 264 (Fig. 5c), which was consistent with previous findings in humans ${ }^{44}$. We also found that enrichment 265 for variants associated with complex traits was significantly positively correlated with signatures 266 of selection (Supplementary Fig. 10b,c). We then asked if tissue-specific REs were involved in 267 genetic control of specific complex traits. To answer this question, we conducted GWAS signal 
268 enrichment analysis for average daily gain (ADG) in three separate breeds (i.e., Duroc, Landrace

269 and Yorkshire), with emphasis on tissue-specific TssA and EnhA. As we expected, muscle,

270 adipose, liver, and gut-common regulatory elements were the most relevant for ADG (Fig. 5d). In

271 further examining the top ADG QTLs in Landrace (Fig. 5e), we found that the top hit SNPs that

272 are within a muscle-specific EnhA (Fig. 5f) that appears to target two genes (ZNF532 and ALPK2)

273 based on TAD and Hi-C loop derived from CTCF and Hi-C data. Among all seven genes within

274 this QTL, ALPK2 plays important roles in cardiogenesis and was upregulated in the longissimus

275 dorsi muscle in Wannanhua compared with Yorkshire pig ${ }^{45,46}$, and was the only gene specifically

276 expressed in muscle (Fig. 5f-h). Additional evidence from the eQTLs in muscle showed the highest

277 enrichment in accessible enhancers (EnhA and EnhAMe) compared to other chromatin states

278 (Supplementary Fig. 10d), suggesting that genetic regulatory variants are more likely to influence

279 gene expression through perturbing enhancers. In summary, these results together demonstrated

280 the important role of functional genome annotation for interpreting the molecular mechanisms

281 underpinning complex traits, adaptive evolution and gene regulation.

\section{Comparative analysis of pig, mouse and human epigenomes}

283 The distribution of individual histone epigenetic marks and chromatin accessibility with respect

284 to genomic features (e.g., 5'UTRs and exons) was consistent between pig, mouse, and human

285 (Supplementary Fig. 11). To determine if the chromatin states are similarly conserved between

286 these species, we predicted 15 chromatin states in mouse and human based on the same epigenetic

287 marks in pig. The resulting chromatin state predications demonstrated general similarity among

288 the three species in terms of genome coverage, genomic distribution and sequence conservation

289 (Fig. 6a, Supplementary Fig. 12). 
To explore the relationship between the epigenome and DNA sequence conservation among 291 three species, we divided each genome into regions corresponding to 50 different levels of 292 sequence conservation $\left(0^{\text {th }}-49^{\text {th }}\right)$ (Methods). Our results revealed that the majority of chromatin 293 states showed higher conservation levels in sequences under both rapid and slow evolution than 294 those under neutral evolution, following a U-shaped distribution ${ }^{47}$ (Fig. 6b, Supplementary Fig. 295 13a). We also found that the densities of chromatin states and gene elements followed the similar 296 U-shaped distribution (Supplementary Fig. 13b,c), supporting the hypothesis that conserved epi297 modifications may buffer negative selective pressures by providing the genome more elastic room 298 to adapt ${ }^{47}$. Furthermore, we categorized orthologous genes into 50 groups based on the degree of 299 conservation of gene expression between species and observed that genes with more conserved 300 expression levels also demonstrated more conserved TssA and TssBiv signatures (Fig. 6c). In 301 further examining sequence extremely conserved $\left(49^{\text {th }}\right)$ or extremely variable regions $\left(0^{\text {th }}\right)$, genes 302 linked to TssA shared by human and pig are involved in basic biological processes, such as ncRNA 303 metabolic process and mRNA catabolic process (Supplementary Fig.14). For the sequence 304 extremely conserved region $\left(49^{\text {th }}\right)$, we found that genes proximal $( \pm 2 \mathrm{~kb})$ by human-specific 305 (comparing with pig) TssA in brain (e.g., FOXG148) are engaged in neuron fate commitment, 306 cerebral cortex development, learning and memory (Fig. 6d, Supplementary Fig. 15, 307 Supplementary Table 10).

308 Next, we evaluated the evolutionary basis of complex traits in humans. Heritability enrichment 309 analysis of 47 complex traits across 15 chromatin states that were mapped from pigs to orthologous 310 regions in humans found that promoters and TSS-proximal transcribed regions were most enriched 311 for variants (Fig. 6e). We further revealed that the more conserved (species-shared) chromatin 312 states showed significantly higher enrichment of complex traits heritability than the more 
313 divergent (species-specific) chromatin states (Fig. 6f). Then we further examined the role of tissue-

314 specific gene regulation on human complex traits. Our heritability enrichment analysis of complex

315 traits, based on human orthologous regions of tissue-specific EnhAs identified in pigs,

316 demonstrated that tissue-specific enhancers were significantly enriched for the corresponding

317 human complex traits relevant to biological functions of specific tissues (Fig. 6g). For instance,

318 the lung-specific EnhAs were significantly enriched for the heritability of lung forced expiratory

319 volume 1 (FEV1), liver for fasting glucose and cholesterol, colon for Crohn's disease, and cortex

320 for intelligence (Fig. 6g).

321 Finally, we sought to determine if this annotation of regulatory elements substantiated the use

322 of pig as an appropriate animal model for different human diseases by comparing human, mouse

323 and pig epigenomes in specific tissues. In brain cortex, the mouse-human shared EnhAs exhibited

324 significantly higher heritability enrichment than the pig-human shared EnhAs for most brain-

325 relevant traits, such as attention deficit hyperactivity disorder (ADHD), intelligence, depression

326 and reaction time, with the exception of Alzheimer's disease, for which heritability was

327 significantly enriched in pig-human shared EnhAs rather than the mouse-human shared EnhAs

328 (Fig. 6h). This was in line with previous findings that pigs have been used as a biomedical model

329 for studying Alzheimer's disease ${ }^{21,49}$. Similar observations were found in intestine (Crohn's disease

330 and inflammatory bowel disease (IBD), but not colorectal cancer, which demonstrated more

331 heritability in the pig-human shared EnhAs) (Fig. 6i) and in adipose (body mass index (BMI),

332 body fat percentage, waist-hip ratio and weight with significantly higher heritability enrichments

333 in the pig-human shared EnhAs) (Fig. 6j). Similar results showed in promoter (TssA)

334 (Supplementary Fig.16). Our findings suggest the pig could be a better biomedical model for

335 certain human traits and diseases than mouse, and vice versa. 


\section{Discussion}

338 In this study, we provided the most comprehensive catalog of porcine regulatory elements to

339 date, spanning 14 tissues including six gut-associated tissues, and characterized the dynamic

340 chromatin state landscape across these tissues and uncovered extensive tissue-specific regulation

341 of gene expression.

342 The annotation of functional elements in human and mouse has proven highly effective for the

343 identification of causative variants of complex traits ${ }^{23,27}$. Our results also demonstrated that variants

344 of complex traits and eQTLs of growth-related traits were significantly enriched in the active

345 promoters and enhancers annotated by this study. Specifically, we speculate that a potential

346 causative SNP, which was associated with average daily gain and which was found within a

347 muscle-specific enhancer, may regulate the expression of $A L P K 2^{45,46}$, a gene demonstrating

348 muscle-specific expression (0.5Mb away). In addition, our annotation of functional elements in

349 pigs allows us to evaluate the potential role of regulatory elements on pig domestication. Our 350 analysis illustrated that signatures of domestication were significantly enriched in porcine

351 regulatory elements. Specifically, genetic variants in the spleen-specific promoters were enriched

352 during Asian pig domestication, whereas variants within cortex-specific promoters were enriched

353 during European pig domestication. This novel insight may reflect the observed distinct

354 phenotypic difference between Asian (more disease resistance ${ }^{41}$ ) and European domesticated pigs

355 (more active and aggressive $\mathrm{e}^{42,43}$ ). Further investigation is warranted to deepen our understanding

356 of genetic selection and domestication in the pig. This regulatory element atlas will serve as a

357 valuable source for the livestock community to inform GWAS and eQTL findings, genomic 
358 selection program, and genome editing strategies, as well as to enhance our understanding of

359 genome evolution and adaptation. With continued efforts by the FAANG Consortium ${ }^{50}$, more

360 epigenomic data will be available from diverse samples such as reproduction related tissues,

361 additional developmental stages, and different physiological states. The systemic integration of

362 "omics" data, for instance, the on-going pig GTEx effort will contribute additional insight into the

363 biological mechanisms that underpin agronomic traits, and thereby enhancing genetic

364 improvement of economically important phenotypes ${ }^{50}$.

365 Finally, this atlas of functional elements provided a unique opportunity for comparative 366 epigenomic analysis between human, mouse and pig, the results of which can inform which species

367 constitute the most appropriate biomedical model(s) for specific human diseases. We observed

368 that regions under positive or negative selective pressure demonstrated higher conservation of

369 epigenetic signatures (such as TssA, TssBiv and TxFlnk) than those under neutral selective

370 pressure, further confirming the hypothesis that elasticity of regulatory conservation may play an

371 important role in the evolution of the less conserved regions (impact of negative selection

372 pressure) $)^{47}$. Recently evolved liver enhancers (i.e., species-specific) are often associated with

373 genes that show evidence for being under positive selection ${ }^{51}$. Such enhancers have been further

374 demonstrated to actively affect gene expression, although they have a lower effect than those

375 enhancers shared across species when the comparison is controlled for number of enhancer

376 elements acting on that gene ${ }^{52}$. However, the human-specific promoters in brain tissues were

377 enriched in intelligence related genes, which suggests a critical role for epigenomic regulation of

378 novel biological function in humans in the sequence most evolutionarily conserved regions. It is

379 widely accepted that neither mouse nor pig is universally appropriate to serve as an animal model

380 for every human disease ${ }^{18,53}$. Gene regulatory networks play significant roles in controlling 
381 phenotypic variance of complex traits, including most human diseases. In examining heritability

382 enrichment of 47 complex traits in humans, our epi-conservation analysis among three species by

383 comparing pig-human vs. mouse-human shared enhancers in different tissues revealed novel

384 insights and potential underlying molecular mechanisms as to why pig might be a more appropriate

385 animal model for certain human diseases than mouse and vice versa. This new line of evidence

386 aligns well with many studies of human diseases using either mouse or pig as an animal model ${ }^{18}$.

387 Our study provides a new basis for understanding genetic regulation of complex traits, such as

388 human diseases by focusing on regulatory network conservation across different mammalian

389 species. While the findings from our study are exciting, they are not yet conclusive. More

390 epigenomic data from additional tissues and cell types, as well as additional species such as non-

391 human primates, along with more experimental studies will be needed to extend and functionally

392 validate these mechanisms that underpin complex traits and diseases ${ }^{9,47}$.

\section{Methods}

\section{Animals and tissues}

397 Procedures for tissue collection followed the Animal Care and Use protocol (\#18464) from the 398 Institutional Animal Care and Use Committee (IACUC), University of California, Davis. We 399 collected five gut-associated tissues (stomach, jejunum, duodenum, ileum, and colon) of two 400 Yorkshire littermate male pigs at six months of age from Michigan State University ${ }^{29}$. Cecum from 401 two female hybrid pigs (Yorkshires X Hampshires, five months) were obtained at University of 402 California, Davis meat lab. Tissues were first flash frozen in liquid nitrogen, and then stored at $40380{ }^{\circ} \mathrm{C}$ until further processing. 


\section{Library construction and sequencing}

405 We performed ChIP-seq (H3K4me3, H3K4me1, H3K27ac and H3K27me3) experiments on 406 flash-frozen tissue samples using the iDeal ChIP-seq kit for histones (Diagenode Cat.\#C01010059, 407 Denville, NJ), as previously described ${ }^{29}$. Libraries were sequenced on the Illumina's HiSeq 4000 408 with 50 bp single-end reads. ATAC-seq libraries were generated from frozen tissue samples by a 409 modified protocol (https://figshare.com/articles/dataset/Final_ATAC_protocol_docx/13891268) 410 according to the protocol of Omni-ATAC ${ }^{54}$ and cryopreserved nuclei ${ }^{55}$. The sequencing was 411 performed on Illumina's NextSeq with paired-end 40 bp reads (PE40). For the RRBS-seq 412 experiments, DNeasy Blood \& Tissue Kit (Qiagen, Hilden, Germany) was used for extraction of 413 DNA from frozen tissues. The samples were sent to Novogene (Sacramento, CA, USA) for library 414 construction and sequencing by Illumina HiSeq 4000 PE150. Total RNA isolated from flash415 frozen tissue by Zymo Quick-RNA ${ }^{\text {TM }}$ Miniprep Kit (Irvine, CA, USA). RNA-seq libraries were 416 constructed by NEBNext ${ }^{\circledR}$ Poly(A) mRNA Magnetic Isolation Module kit (NEB \#E7490) and 417 NEBNext巴 Ultra ${ }^{\text {TM }}$ Directional RNA Library Prep Kit for Illumina (NEB \#E7720, New England 418 Biolabs (NEB), Ipswich, MA) and sequenced on Illumina HiSeq 4000 with PE100.

\section{Data processing and data summary}

420 In total, 95 new datasets including ChIP-seq (H3K4me3, H3K4me1, H3K27ac, H3K27me3, 421 input control), ATAC-seq, RRBS, RNA-seq in two biological replicates of six gut-associated 422 tissues were generated. We also integrated additional 144 existing pig epigenomic datasets 423 including ChIP-seq (H3K4me3, H3K4me1, H3K27ac, H3K27me3, CTCF, input control), ATAC424 seq, RRBS, RNA-seq in the same two biological replicates of eight core tissues (Adipose, 425 Cerebellum, Cortex, Hypothalamus, Liver, Lung, Muscle, Spleen) from our FAANG pilot project 426 (PRJEB14330) ${ }^{29}$, and four Hi-C pig liver datasets from publicly available dataset (PRJEB27364) ${ }^{30}$. 
427 UC Davis FAANG Functional Annotation Pipeline (https://github.com/kernco/functional428 annotation) was applied to process the ChIP-seq, ATAC-seq, and RNA-seq as previously 429 described $^{29}$. Briefly, the susScr11 genome assembly and Ensembl genome annotation (v100) were

430 used as references for pig. Sequencing reads were trimmed with Trim Galore! ${ }^{56}(\mathrm{v} .0 .6 .5)$, and 431 aligned with $\operatorname{STAR}^{57}(\mathrm{v} \cdot 2.5 .4 \mathrm{a})$ or $\mathrm{BWA}^{58}(\mathrm{v} 0.7 .17)$ to the respective genome assemblies. 432 Alignments with MAPQ scores less than 30 were filtered using Samtools ${ }^{59}$ (v.1.9). For RNA-seq, 433 gene counts were determined using htseq-count ${ }^{60}(\mathrm{v} \cdot 0.13 .5)$, and then trimmed mean of M-values 434 (TMM) and transcript per million (TPM) normalization were performed using EdgeR (v3.32.0) 435 and StringTie2 (v.1.3.3), respectively ${ }^{61}$. For ChIP-seq, after the filtering, duplicates were marked 436 and removed using Picard (v.2.18.7). Regions of signal enrichment ("peaks") were called by 437 MACS2 ${ }^{62}$ (v.2.1.1). Various quality metrics (e.g., JSD, Supplementary Table 1) were calculated 438 following the method described in our previous study ${ }^{29}$. RRBS data were processed using 439 Bismark ${ }^{63}$ (v.0.22.1) with parameter set in RRBS pipeline (https://github.com/zhypan/Functional440 Annotation-of-Pig). We called the Hi-C contacts using the Juicer pipeline ${ }^{64}$ with default parameters. 441 The global correlations among assays, tissues, and biological replicates were performed by 442 deepTools ${ }^{65}$ (v.3.5.0). Briefly, the Z-score normalized read signals of all samples with step of $1 \mathrm{k}$ 443 bp window were calculated by multiBigwigSummary and were presented by plotCorrelation. The 444 signal of marks along with protein coding genes were generated by deepTools ${ }^{65}$ (computeMatrix 445 scale-regions function) with parameter -a 2500 -b 2500. The Z-score was used to normalize bigWig 446 of five marks as input files.

\section{$447 \quad$ Annotation of chromatin states}

448 ChromHMM $^{66}$ (v.1.20) was used to train the chromatin state prediction model by integrating 449 ChIP-seq (H3K4me3, H3K4me1, H3K27ac, H3K27me3, and input control) and ATAC-seq data 
450 from two biological replicates of 14 tissues. The same tissue of two biological replicates were 451 collectively considered as one tissue epigenome. The 15-state model was chosen, as it presented 452 maximum number of states with distinct epigenetic mark combinations. We labelled these 15 453 chromatin states based on their combinations of histone modifications and enrichment around $454 \mathrm{TSS}^{8,27}$. Then the fold enrichment of each chromatin state for each external gene element (e.g., 455 exon, $\mathrm{CpG}$ islands) was calculated by $(\mathrm{C} / \mathrm{A}) /(\mathrm{B} / \mathrm{D})$, where $\mathrm{A}, \mathrm{B}, \mathrm{C}, \mathrm{D}$ are the number of bases in a 456 chromatin state, a gene element, overlapped between a chromatin state and a gene element, in the

457 genome, respectively. In addition, we also computed chromatin state fold enrichment in 458 mammalian conserved elements which identified from Multiple Sequence Alignments (MSA) 459 using the Genomic Evolutionary Rate Profiling (GERP) software based on 103 mammals 460 (ftp://ftp.ensembl.org/pub/release-100/bed/ensembl-

461 compara/103_mammals.gerp_constrained_element/). The methylation level of each state and its 462 up- and down- stream 10kb was calculated by the computeMatrix scale-regions function of 463 deepTools with parameters --binSize 500, --regionBodyLength 2000 and --skipZeros.

\section{Clustering of large-scale chromatin structure}

465 To examine genome-wide chromatin structure, we first divided the genome (excluding chrUn) 466 into 1,224 fragments of $2 \mathrm{Mb}$ in length. Then we calculated the state frequencies (state bin/total $467 \mathrm{bin}$ ) in each $2 \mathrm{Mb}$ fragment for each tissue and then the average frequency across tissues. To 468 identify modules, column clustering was performed by k-means $=12$, and rows were clustered using $469 \mathrm{k}=3$. In addition, we calculated number of protein-coding, lncRNA, and $\mathrm{CpG}$ islands for each $2 \mathrm{Mb}$ 470 fragment by BEDTools ${ }^{67}$ (v.2.29.2). We also calculated the average TPM of protein coding gene 471 and average methylation level across 14 tissues in each $2 \mathrm{Mb}$ fragment. Then the average gene 
472 expression and methylation level in each of the 12 modules were calculated and a Student's T-test

473 was performed with parameter setting ref.group = "M3".

\section{Chromatin state variability}

475 For each state, we first obtained regulatory regions across 14 tissues (RRATs) (Supplementary

476 Fig. 4a-c) using BEDtools merge function (any regulatory region between two tissues overlapped

477 by 1 bp was merged), then we calculated the total genomic length for each tissue (GL) and the

478 total combined genomic length (TGL) for RRATs. The relative state coverage per tissue was

479 derived by GL/ TGL (Supplementary Fig. 4d). Finally, followed by the order from high to low

480 based on the GL/TGL value in each tissue, we calculated the total genomic length of accumulated

481 tissues (aGL) by adding one tissue each time until all 14 tissues were added, and the cumulative

482 state coverage was calculated as aGL/ TGL. States whose cumulative coverage changed faster than

483 others were considered to be less constitutive (more variable) states.

\section{Chromatin state switching between tissues}

485 Chromatin state switching between tissues was calculated by pairing two tissues. Given a 486 pairing of A and B tissues, we first counted total bins of chromatin state "e" in A (TbAe), then 487 obtained the overlap bins of chromatin state "e" (Obe) in A and B, then computed the state 488 switching probabilities using Obe/TbAe for the tissue A to B transition and Obe/TbBe for the 489 tissue B to A transition. By averaging these calculations for a pair of tissues, we obtained the pair 490 switching probabilities. We calculated the state switching probabilities in between intestinal 491 tissues, between brain tissues (Supplementary Fig. 6a,b) and between 8 distinguishable tissues 492 (jejunum, cortex, adipose, liver, lung, muscle, spleen). 


\section{Hierarchical epigenome clustering}

494 We first calculated the an epigenetic mark's signal confidence scores $\left(-\log _{10}\right.$ (Poisson $P$ value $\left.)\right)$ 495 within 200 bp of the genomic regions for each mark of each sample as described in 496 http://jvanheld.github.io/stats_avec_RStudio_EBA/practicals/02_peak-calling/peak-

497 calling_report.html\#data_sets. Then, we extracted a specific mark's signal confidence score of 498 each sample for specific state of RRATs regions. For example, we extract H3K4me1 signal 499 confidence scores for EnhA. After combining all samples' mark confidence scores for each tissue 500 and each state, we constructed a distance matrix using the ward.D2-linkage hierarchical clustering 501 following by Euclidean distance method in R.

\section{Promoter enrichment analysis of tissue-specific expressed genes among 14 tissues}

503 To evaluate how chromatin state changes at promoter regions of TSE genes across tissues, we 504 first performed a Student's $t$-test among 14 tissues to identify tissue-specific expressed genes based 505 on TPM. We further grouped some tissues into different sub-groups such as small intestine 506 (Jejunum, Ileum, Duodenum), large intestine (Cecum, Colon), and brain (Cortex, Cerebellum, 507 Hypothalamus), and identified tissue-specific expressed genes by excluding the tissues in the same 508 sub-group. Then we selected genes with the top 5\% t-value as TSE genes ${ }^{68}$. The biological process 509 of GO enrichment for these TSE genes were identified by WebGestalt $2019^{69}$ using the default 510 significance level $(\mathrm{FDR}<0.05)$. Then we calculated the chromatin state fold enrichment of TSE

511 (up and down stream 2000bp around TSS) in each tissue and the change in enrichment by TSE 512 enrichment in specific tissue minus other tissues.

\section{Chromatin state switching of target enhancer (EnhA) of TSE gene}


514 To evaluate how enhancers of TSE genes switch among tissues, we first identified the target

515 enhancers of TSE genes following the method described in our recent study ${ }^{29}$. Briefly, we

516 generated the predicted TADs from CTCF ChIP-seq data by FIMO ${ }^{70}$ following the method

517 described in Oti, et al. ${ }^{71}$. Then we predicted the enhancer-gene pairs according to the Spearman's

518 rank correlation of every possible combination of regulatory element H3K27ac signal and gene

519 expression value within each TAD. Benjamini-Hochberg adjustment (FDR < 0.05$)$ was used to

520 define putative interacting pairs. The enhancers in the enhancer-gene pairs that corresponded to

521 TSE genes were considered as TSE genes' target enhancers. Finally, we computed enhancer state

522 switching probabilities of TSE genes among tissues using the method described above.

\section{TSR of enhancer, promoter and their putative functional regulation}

524 For strong enhancer (EnhA) identified in each tissue, we counted the bins of overlapping RRATs

525 by comparing to other tissues. If the number of bins $>=1$, the tissue of this RRATs region would

526 be assigned 1, otherwise it was assigned 0 . We generated a total of 17 modules of tissue-specific

527 regulatory elements (TSR) enhancers. The 17 modules included all-common (presented in all

528 tissues), gut-common (presented in all 5 intestinal tissues), brain-common (presented in all 3 brain

529 tissues) and 14 tissue-specific modules. The same method was used to obtain TSR for promoters

530 (1_TssA). In addition, we performed enrichment analyses (GO, Human Phenotype Ontology

531 (HPO), Mouse Phenotype) based on genes proximal to TSR using the GREAT ${ }^{72}$ tool with default

532 parameters except for TSR promoters (proximal $2 \mathrm{~kb}$ upstream, $1 \mathrm{~kb}$ downstream, plus distal up to

$5333 \mathrm{~kb})$. We used a cut-off of FDR $<0.05$ for both the binomial and the hypergeometric distribution-

534 based tests. 
535 The motifs of tissue-specific EnhAs were identified by HOMER $^{73}$ (v.4.11) with cutoff

536 FDR $<0.05$. We selected the top three enriched or tissue function relevant motifs for each tissue as

537 the candidate tissue-specific EnhAs motifs and generated a total of 51 motifs enriched in tissue-

538 specific EnhAs. In addition, we used these 51 motifs as known TF motifs to conduct the enrichment

539 for all tissues by HOMER. The mRNA expression of corresponding TFs in pigs were used to

540 calculate the correlation with motif enrichment.

\section{Selection signature enrichment analysis of chromatin state}

542 A total of 406 whole genome sequence datasets (Supplementary Table 8 ) in pigs (Asian wild

543 (58) and domestic pigs (129), European wild (35) and domestic pigs (184)) were trimmed by 544 Trimmomatic ${ }^{74}$ (v.0.39), mapped by BWA (0.7.17), and marked duplicates by GATK ${ }^{75}$ (v4.1.4.1)

545 MarkDuplicates with default parameters. The SNPs of Gvcf for each sample were called by GATK

546 HaplotypeCaller. All Gvcf were then combined and the variants for each sample were called by

547 GenotypeGVCFs. After SNP calling, the variants were filtered using VariantFiltration $(\mathrm{QD}<2.0$,

$548 \mathrm{MQ}<40.0, \mathrm{FS}>60.0, \mathrm{SOR}>3.0$, MQRankSum $<-12.5$, ReadPosRankSum < -8.0) to remove

549 low-quality SNPs. We then performed Fst analysis between Asian wild and domestic pigs, and

550 between European wild and domestic pigs, and calculated the fold enrichment of selection 551 signature for chromatin states using the same method for gene elements enrichment described 552 above.

\section{GWAS and eQTL signal enrichment of chromatin state}

554 The pig GWAS data of 44 traits was described previously ${ }^{76,77}$ (Supplementary Table 9). First, we 555 filtered out all SNPs with minor allele frequency below $0.5 \%$, with a large deviation from Hardy- 
556 Weinberg proportions $\left(P<1.0^{-6}\right)$, or with a R2 value of the imputation accuracy estimated by

557 Minimac4 of less than 0.4. We performed GWAS signal enrichment of 44 pig complex traits (3

558 daily gain related, 20 lipid related, and 21 feed efficiency related) for each chromatin state across

55914 tissues using a 10,000 times genotype cyclical permutation tests ${ }^{68}$. The eQTLs data in pig

560 muscle $^{78}$ with FDR $<0.05$ were used to calculate the fold enrichment for the chromatin states using

561 the same method above.

\section{Interspecies conservation of chromatin state}

563 We collected data from ENCODE ${ }^{4,9}$, Roadmap Epigenomics $^{8}$ and published $\operatorname{articles}^{79}(9$ tissues

564 in human and 7 tissues in mouse, Supplementary Table 11,12), including ChIP-seq (H3K4me3,

565 H3K4ac, H3K4me1, H3K27me3, Input), ATAC-seq, DNase-seq, and RNA-seq. In total, we

566 obtained six matched tissues (small intestine, liver, spleen, lung, adipose, cortex) among pig,

567 human, and mouse. All the data were processed following the same pipeline used in pig. The

568 GRCh38 (human) and GRCm38 (mouse) assemblies with Ensembl annotations (v100) were used

569 for data analysis. Chromatin states of human and mouse were also trained by ChromHMM and 15

570 chromatin states were identified. To explore the relationship between sequence conservation and

571 epi-conservation among the three mammals, we first divided the genome into 50 equal sized sets

$572\left(0^{\text {th }}-49^{\text {th }}\right)$ with increasing average PhyloP scores using the method detailed by Xiao et $a^{47}$. Briefly,

573 the human genome was divided into 15 million 200 bp segments. Then average PhyloP score (100

574 vertebrate genomes ${ }^{80}$ ) was computed for each 200 bp segment. These genomic segments were

575 divided into 50 equal sized sets from the fastest changing sequence (smallest PhyloP scores) to the

576 most conserved (greatest PhyloP scores). (Supplementary Fig. 13d). To quantify epigenomic

577 conservation, we downloaded the whole genome alignments UCSC chain files among human 
578 (hg38), pig (SusScr11), mouse (mm10) and processed as described in the UCSC Genome Wiki 579 website (http://genomewiki.ucsc.edu/index.php/HowTo:_Syntenic_Net_or_Reciprocal_Best) to

580 derive reciprocal best chains. Then we converted genomic coordinates between assemblies using

581 the UCSC Liftover tool (https://genome.sph.umich.edu/wiki/LiftOver) based on 0.65 sequence

582 identity. All the chromatin states in pig and mouse were lifted over to human. The conservation

583 rate $(0 \sim 1)$ of each region of each state from pig to human was calculated based on state region

584 coverage of pig over human. If there was no overlap it was assigned 0 , if completely occupied it

585 was assigned 1. The same analysis was conducted for pig to mouse and mouse to human.

586 Furthermore, we performed genomic and epigenomic conservations for every pair of mammalian

587 species in each tissue. Finally, we conducted the same analysis on mammalian conserved score

588 based Genomic Evolutionary Rate Profiling (GERP) using 103 mammalian genomes

589 (ftp://ftp.ensembl.org/pub/release-

590 100/compara/conservation_scores/103_mammals.gerp_conservation_score/)

591 To examine the biological relevance of sequence extremely variable $\left(0^{\text {th }}-2^{\text {th }}\right.$ sets $)$ and conserved 592 regions $\left(47^{\text {th }}-49^{\text {th }}\right.$ sets), we extracted the human-pig shared and human-specific chromatin state 593 TssA from these regions. Then the GREAT tool with parameter of proximal $2 \mathrm{~kb}$ upstream, $1 \mathrm{~kb}$ 594 downstream, plus distal up to $3 \mathrm{~kb}$ was used to conduct GO function enrichment analysis.

\section{Expression conservation versus epi-conservation}

596 The TPM of 14302 orthologous genes from pig, human, and mouse were used to identify 597 differentially expressed genes in each tissue using the Student's $t$-test. We sorted the genes by p598 value within each species and divided them into 50 equally sized sets. Then we calculated the 599 average epi-conservation score of states in the 20kb region around TSS of gene in each set. 


\section{Heritability enrichment of human complex traits in chromatin state}

601 To explore how conserved or species-specific chromatin states affects complex traits in humans,

602 we extracted six types of species-share or species-specific regulatory elements (all_shared, 603 human_mouse_shared, human_pig_shared, human_specific, mouse_specific, pig_specific). We

604 applied stratified linkage disequilibrium score regression (LDSC) to partition heritability of 47

605 human complex traits into distinct functional categories ${ }^{44}$, which revealed which functional regions

606 explained more genetic variation of complex traits from an evolutionary point of view. These

607 functional categories included six types of species-shared/specific regulatory elements, chromatin

608 state of each tissue, and TSR of EnhA/TssA. We calculated the stratified LD scores using 1000G

609 Phase 3 European human samples, where only HapMap3 SNPs with INFO $\geq 0.9$ and MAF $>0.05$ in

610 1000G European samples were used (the 1000G samples and default SNP weights were obtained

611 from https://github.com/bulik/ldsc).

612 The GWAS summary statistics for 47 human complex traits were obtained from public

613 databases (Supplementary Table 13), with an average sample size of 321,978 (all European

614 ancestry) and a high quality overlap with HapMap3 panel. In addition, these GWAS results have,

615 a mean $\chi^{\wedge} 2$ statistics of $>1.02$ and a heritability Z-score of $>4^{81}$. We also performed default

616 quality control for each GWAS summary statistics by LDSC to remove GWAS SNPs that are with

$617 \mathrm{MAF} \leq 0.01$, genotype call rate $\leq 0.75$, INFO $\leq 0.9$, out-of-bounds $P$-value, duplicated Rsid, strand

618 ambiguous variants and extreme large $\chi^{\wedge} 2$ statistics $^{81}$. The results of LDSC regression for base

619 model, which has not been partitioned heritability, are available in Supplementary Table 14. 


\section{Data availability}

622 All high-throughput sequencing data in this study were deposited in European Nucleotide 623 Archive (ENA) with accession number PRJEB37735 and PRJEB14330. All raw data are also

624 available through the FAANG portal (https://data.faang.org/dataset). All processed data are

625 publicly available at https://doi.org/10.6084/m9.figshare.13480425. Chromatin states of pig, 626 mouse, and human are available in Genome Browser:

627 http://genome.ucsc.edu/s/zhypan/susScr11_15_state_14_tissues_new

628 http://genome.ucsc.edu/s/zhypan/mm10 7tissues chr state;

629 http://genome.ucsc.edu/s/zhypan/hg38_9tissue_chr_state.

\section{Code availability}

631 The pipeline for RNA-seq, ATAC-seq, DNase-seq and ChIP-seq processing is available at 632 https://github.com/kernco/functional-annotation. RRBS pipeline and other processing codes are 633 publicly available at https://github.com/zhypan/Functional-Annotation-of-Pig.

\section{Acknowledgements}

636 This study was supported by Agriculture and Food Research Initiative Competitive Grant no. 637 2018-67015-27501 (CKT, HZ CE and PR) and no. 2015-67015-22940 (HZ and PR) from the 
638 USDA National Institute of Food and Agriculture, Multistate Research Project NRSP8 and

639 NC1170 (HZ), and the California Agricultural Experimental Station (HZ).

645 Author contributions

647 H.Z., L.F. Z.P. P.R., C.W. E. and C.K.T. conceived and designed the study. C.E., Y.W., K.C.

648 and Z.P. were responsible for sample collection. Z.P., Y.W. and M.H. performed ChIP-seq,

649 ATAC-seq, RNA-seq. Z.P., N.T. and K.W. contribute for RRBS data collection. Z.P., Y.Y. L.F.

650 and C. K. conducted bioinformatic analysis. Z.X., G.S., GS. S., MS. L., M.F., and P. KM. were

651 responsible for pig GWAS data analysis. H.Y. and L.B. responsible for pig selection signature

652 collection. Z.P., L.F., Y.Y. and H.Z. wrote the initial draft of the manuscript. M.H. C.W. E., P.

653 R., and C.K. T. revised manuscript. All co-authors contributed to the final manuscript.

\section{Competing interests}

656 The authors declare no competing interests. 


\section{Additional information}

660 Correspondence and requests for materials should be addressed to H.Z., and L.F.

661 Reprints and permissions information is available at www.nature.com/reprints.

662

663 References

664

665 1. Consortium, E.P. et al. Expanded encyclopaedias of DNA elements in the human and 666 mouse genomes. Nature 583, 699-710 (2020).

667 2. Consortium, E.P. The ENCODE (ENCyclopedia of DNA elements) project. Science 306, $668 \quad$ 636-640 (2004).

$6693 . \quad$ Consortium, E.P. Identification and analysis of functional elements in $1 \%$ of the human 670 genome by the ENCODE pilot project. Nature 447, 799 (2007).

671 4. Dunham, I. et al. An integrated encyclopedia of DNA elements in the human genome. $672 \quad$ Nature 489, 57 (2012).

673 5. Filion, G.J. et al. Systematic protein location mapping reveals five principal chromatin 674 types in Drosophila cells. Cell 143, 212-224 (2010).

675 6. Ernst, J.et al. Mapping and analysis of chromatin state dynamics in nine human cell 676 types. Nature 473, 43-49 (2011). 
677 7. Pang, B. \& Snyder, M.P. Systematic identification of silencers in human cells. Nat.

$678 \quad$ Genet. 52, 254-263 (2020).

679 8. Roadmap Epigenomics, C. et al. Integrative analysis of 111 reference human

$680 \quad$ epigenomes. Nature 518, 317-330 (2015).

6819 9. Gorkin, D.U. et al. An atlas of dynamic chromatin landscapes in mouse fetal

682 development. Nature 583, 744-751 (2020).

683 10. Zabidi, M.A. et al. Enhancer-core-promoter specificity separates developmental and 684 housekeeping gene regulation. Nature 518, 556-559 (2015).

685 11. He, Y. et al. Spatiotemporal DNA methylome dynamics of the developing mouse fetus.

$686 \quad$ Nature 583, $752-759$ (2020).

687 12. Roy, S. et al. Identification of functional elements and regulatory circuits by Drosophila 688 modENCODE. Science 330, 1787-1797 (2010).

689 13. Gerstein, M.B. et al. Integrative analysis of the Caenorhabditis elegans genome by the 690 modENCODE project. Science 330, 1775-1787 (2010).

691 14. Maurano, M.T. et al. Systematic localization of common disease-associated variation in 692 regulatory DNA. Science 337, 1190-1195 (2012).

693 15. Boix, C.A., James, B.T., Park, Y.P., Meuleman, W. \& Kellis, M. Regulatory genomic 694 circuitry of human disease loci by integrative epigenomics. Nature, 1-8 (2021).

695 16. Zhang, Q., Widmer, G. \& Tzipori, S. A pig model of the human gastrointestinal tract. Gut $696 \quad$ Microbes 4, 193-200 (2013).

697 17. Bassols, A. et al. The pig as an animal model for human pathologies: A proteomics 698 perspective. PROTEOMICS-Clinical Applications 8, 715-731 (2014). 
699 18. Meurens, F., Summerfield, A., Nauwynck, H., Saif, L. \& Gerdts, V. The pig: a model for 700 human infectious diseases. Trends Microbiol. 20, 50-57 (2012).

701 19. Sullivan, T.P., Eaglstein, W.H., Davis, S.C. \& Mertz, P. The pig as a model for human 702 wound healing. Wound Repair Regen. 9, 66-76 (2001).

703 20. Gieling, E.T., Schuurman, T., Nordquist, R.E. \& van der Staay, F.J. The pig as a model 704 animal for studying cognition and neurobehavioral disorders. in Molecular and Functional Models in Neuropsychiatry 359-383 (Springer Press, 2011).

706 21. Kragh, P.M. et al. Hemizygous minipigs produced by random gene insertion and 707

22. de Almeida, A.M. \& Bendixen, E. Pig proteomics: a review of a species in the crossroad between biomedical and food sciences. Journal of Proteomics 75, 4296-4314 (2012).

711 23. Xiang, R. et al. Quantifying the contribution of sequence variants with regulatory and evolutionary significance to 34 bovine complex traits. Proc. Natl. Acad. Sci. USA 116, 19398-19408 (2019).

714 24. Andersson, L. et al. Coordinated international action to accelerate genome-to-phenome with FAANG, the Functional Annotation of Animal Genomes project. Genome Biol. 16,

717 25. Burns, E.N. et al. Generation of an equine biobank to be used for Functional Annotation of Animal Genomes project. Anim. Genet.49, 564-570 (2018).

719 26. Kingsley, N. et al. Functionally annotating regulatory elements in the equine genome using histone mark chip-seq. Genes 11, 3 (2020). 
721 27. Fang, L. et al. Functional annotation of the cattle genome through systematic discovery 722 and characterization of chromatin states and butyrate-induced variations. BMC Biol. 17, $723 \quad 68(2019)$.

724 28. Halstead, M.M. et al. A comparative analysis of chromatin accessibility in cattle, pig, and 725 mouse tissues. BMC Genomics 21, 1-16 (2020).

726 29. Colin Kern, Y.W., Xiaoqin Xu, Zhangyuan Pan, Michelle Halstead, Kelly Chanthavixay, 727 Perot Saelao, Susan Waters, Ruidong Xiang, Amanda Chamberlain, Ian Korf, Mary E. Delany, Hans H. Cheng, Juan F. Medrano, Alison L. Van Eenennaam, Chris K. Tuggle, Catherine Ernst, Paul Flicek, Gerald Quon, Pablo Ross, Huaijun Zhou. Functional

732 30. Foissac, S. et al. Multi-species annotation of transcriptome and chromatin structure in 733 domesticated animals. BMC Biol. 17, 1-25 (2019).

734 31. Tyska, M.J. et al. Myosin-1a is critical for normal brush border structure and 735 composition. Mol. Biol. Cell 16, 2443-2457 (2005).

736 32. Shifrin Jr, D.A. et al. Enterocyte microvillus-derived vesicles detoxify bacterial products

738 33. Wagner, J.R. et al. The relationship between DNA methylation, genetic and expression inter-individual variation in untransformed human fibroblasts. Genome Biol. 15, R37

741 34. Roy, S. et al. Villin-1 and gelsolin regulate changes in actin dynamics that affect cell survival signaling pathways and intestinal inflammation. Gastroenterology 154, 14051420. e1402 (2018). 
744 35. Wutz, A. Gene silencing in X-chromosome inactivation: advances in understanding 745 facultative heterochromatin formation. Nat. Rev. Genet. 12, 542-553 (2011).

746 36. Savilahti, E., Reunala, T. \& Mäki, M. Increase of lymphocytes bearing the gamma/delta 747 T cell receptor in the jejunum of patients with dermatitis herpetiformis. Gut 33, 206-211 $748 \quad$ (1992).

749 37. Hommes, D. et al. Inhibition of stress-activated MAP kinases induces clinical 750 improvement in moderate to severe Crohn's disease. Gastroenterology 122, 7-14 (2002).

751 38. Chen, L. et al. HNF4 regulates fatty acid oxidation and is required for renewal of 752 intestinal stem cells in mice. Gastroenterology 158, 985-999. e989 (2020).

753 39. Silberg, D.G., Swain, G.P., Suh, E.R. \& Traber, P.G. Cdx1 and cdx2 expression during 754 intestinal development. Gastroenterology 119, 961-971 (2000).

755 40. Mach, N. et al. Extensive expression differences along porcine small intestine evidenced 756 by transcriptome sequencing. PLoS ONE 9, e88515 (2014).

757 41. Chen, H. et al. Introgression of Eastern Chinese and Southern Chinese haplotypes 758 contributes to the improvement of fertility and immunity in European modern pigs. $759 \quad$ GigaScience 9 , giaa014 (2020).

760 42. Chu, Q., Liang, T., Fu, L., Li, H. \& Zhou, B. Behavioural genetic differences between $761 \quad$ Chinese and European pigs. J. Genet. 96, 707-715 (2017).

762 43. Li, L.-A. et al. Erhualian and Pietrain pigs exhibit distinct behavioral, endocrine and 763 biochemical responses during transport. Livestock Science 113, 169-177 (2008).

764 44. Finucane, H.K. et al. Partitioning heritability by functional annotation using genome765 wide association summary statistics. Nat. Genet. 47, 1228 (2015). 
766 45. Hofsteen, P. et al. ALPK2 promotes cardiogenesis in zebrafish and human pluripotent 767 stem cells. iScience 2, 88-100 (2018).

768 46. Li, X.J., Zhou, J., Liu, L.Q., Qian, K. \& Wang, C.L. Identification of genes in 769 longissimus dorsi muscle differentially expressed between Wannanhua and Yorkshire 770 pigs using RNA-sequencing. Anim. Genet. 47, 324-333 (2016).

771 47. Xiao, S. et al. Comparative epigenomic annotation of regulatory DNA. Cell 149, 1381$7721392(2012)$.

773 48. Kumamoto, T. \& Hanashima, C. Evolutionary conservation and conversion of Foxg1

774 function in brain development. Development, growth \& differentiation 59, 258-269 $775 \quad$ (2017).

776 49. Lee, S.-E. et al. Production of transgenic pig as an Alzheimer's disease model using a 777 multi-cistronic vector system. PLoS ONE 12, e0177933 (2017).

778 50. Clark, E.L. et al. From FAANG to fork: application of highly annotated genomes to 779 improve farmed animal production. Genome Biol. 21, 1-9 (2020).

780 51. Villar, D. et al. Enhancer evolution across 20 mammalian species. Cell 160, 554-566 $781 \quad$ (2015).

782 52. Berthelot, C., Villar, D., Horvath, J.E., Odom, D.T. \& Flicek, P. Complexity and 783 conservation of regulatory landscapes underlie evolutionary resilience of mammalian 784 gene expression. Nature ecology \& evolution 2, 152-163 (2018).

785 53. Peters, L.L. et al. The mouse as a model for human biology: a resource guide for complex 786 trait analysis. Nat. Rev. Genet. 8, 58-69 (2007).

787 54. Corces, M.R. et al. An improved ATAC-seq protocol reduces background and enables 788 interrogation of frozen tissues. Nat. Methods 14, 959-962 (2017). 
789 55. Halstead, M. et al. Systematic alteration of AtAc-seq for profiling open chromatin in $790 \quad$ cryopreserved nuclei preparations from livestock tissues. Sci.Rep. 10, 1-12 (2020).

791 56. Krueger, F. Trim Galore!: A wrapper tool around Cutadapt and FastQC to consistently 792 apply quality and adapter trimming to FastQ files. (2015).

793 57. Dobin, A. et al. STAR: ultrafast universal RNA-seq aligner. Bioinformatics 29, 15-21 $794 \quad$ (2012).

795 58. Li, H. Aligning sequence reads, clone sequences and assembly contigs with BWA-MEM. 796 arXiv 1303.3997(2013).

797 59. Li, H. et al. The Sequence Alignment/Map format and SAMtools. Bioinformatics 25, $798 \quad$ 2078-2079 (2009).

799 60. Anders, S., Pyl, P.T. \& Huber, W. HTSeq-a Python framework to work with high800 throughput sequencing data. Bioinformatics 31, 166-169 (2014).

801 61. Kovaka, S. et al. Transcriptome assembly from long-read RNA-seq alignments with 802 StringTie2. Genome Biol. 20, 1-13 (2019).

803 62. Zhang, Y. et al. Model-based analysis of ChIP-Seq (MACS). Genome Biol. 9, 1-9 (2008).

804 63. Krueger, F. \& Andrews, S.R. Bismark: a flexible aligner and methylation caller for 805 Bisulfite-Seq applications. Bioinformatics 27, 1571-1572 (2011).

806 64. Durand, N.C. et al. Juicer provides a one-click system for analyzing loop-resolution Hi-C 807 experiments. Cell systems 3, 95-98 (2016).

808 65. Ramírez, F. et al. deepTools2: a next generation web server for deep-sequencing data $809 \quad$ analysis. Nucleic Acids Res. 44, W160-W165 (2016).

810 66. Ernst, J. \& Kellis, M. ChromHMM: automating chromatin-state discovery and 811 characterization. Nat.Methods 9, 215-216 (2012). 
812 67. Quinlan, A.R. BEDTools: the Swiss-army tool for genome feature analysis. Curr.

$813 \quad$ Protoc. Bioinformatics 47, 11.12.11-11.12. 34 (2014).

814 68. Fang, L. et al. Comprehensive analyses of 723 transcriptomes enhance genetic and 815 biological interpretations for complex traits in cattle. Genome Res. 30, 790-801 (2020).

816 69. Liao, Y., Wang, J., Jaehnig, E.J., Shi, Z. \& Zhang, B. WebGestalt 2019: gene set analysis 817 toolkit with revamped UIs and APIs. Nucleic Acids Res. 47, W199-W205 (2019).

818 70. Grant, C.E., Bailey, T.L. \& Noble, W.S. FIMO: scanning for occurrences of a given 819 motif. Bioinformatics 27, 1017-1018 (2011).

820 71. Oti, M., Falck, J., Huynen, M.A. \& Zhou, H. CTCF-mediated chromatin loops enclose 821 inducible gene regulatory domains. BMC Genomics 17, 252 (2016).

822 72. McLean, C.Y. et al. GREAT improves functional interpretation of cis-regulatory regions. $823 \quad$ Nat. Biotechnol. 28, 495-501 (2010).

824 73. Heinz, S. et al. Simple combinations of lineage-determining transcription factors prime 825 cis-regulatory elements required for macrophage and B cell identities. Mol. Cell 38, 576$826 \quad 589(2010)$.

827 74. Bolger, A.M., Lohse, M. \& Usadel, B. Trimmomatic: a flexible trimmer for Illumina 828 sequence data. Bioinformatics 30, 2114-2120 (2014).

829 75. Van der Auwera, G.A. et al. From FastQ data to high-confidence variant calls: the 830 genome analysis toolkit best practices pipeline. Curr. Protoc. Bioinformatics 43, 11.10. $831 \quad 11-11.10 .33(2013)$.

832 76. Zexi Cai, O.F.C., Mogens Sandø Lund, Tage Ostersen, Goutam Sahana. Large-scale 833 association study on daily weight gain in pigs reveals overlap of genetic factors for 834 growth in humans. Genetics Selection Evolution (2021). 
835 77. Zhang, Q. et al. Inclusion of endophenotypes in a standard GWAS facilitate a detailed

836 mechanistic understanding of genetic elements that control blood lipid levels. Sci. Rep.

$837 \quad \mathbf{1 0}, 1-14(2020)$.

838 78. Velez-Irizarry, D. et al. Genetic control of longissimus dorsi muscle gene expression

839 variation and joint analysis with phenotypic quantitative trait loci in pigs. BMC Genomics

$840 \quad 20,3(2019)$.

841 79. Liu, C. et al. An ATAC-seq atlas of chromatin accessibility in mouse tissues. Scientific

$842 \quad$ data 6, 1-10 (2019).

843 80. Pollard, K.S., Hubisz, M.J., Rosenbloom, K.R. \& Siepel, A. Detection of nonneutral

844 substitution rates on mammalian phylogenies. Genome Res. 20, 110-121 (2010).

845 81. Bulik-Sullivan, B.K. et al. LD Score regression distinguishes confounding from

846 polygenicity in genome-wide association studies. Nat. Genet. 47, 291-295 (2015).

847

848

849

850

851

852

853

854

855

856

857 

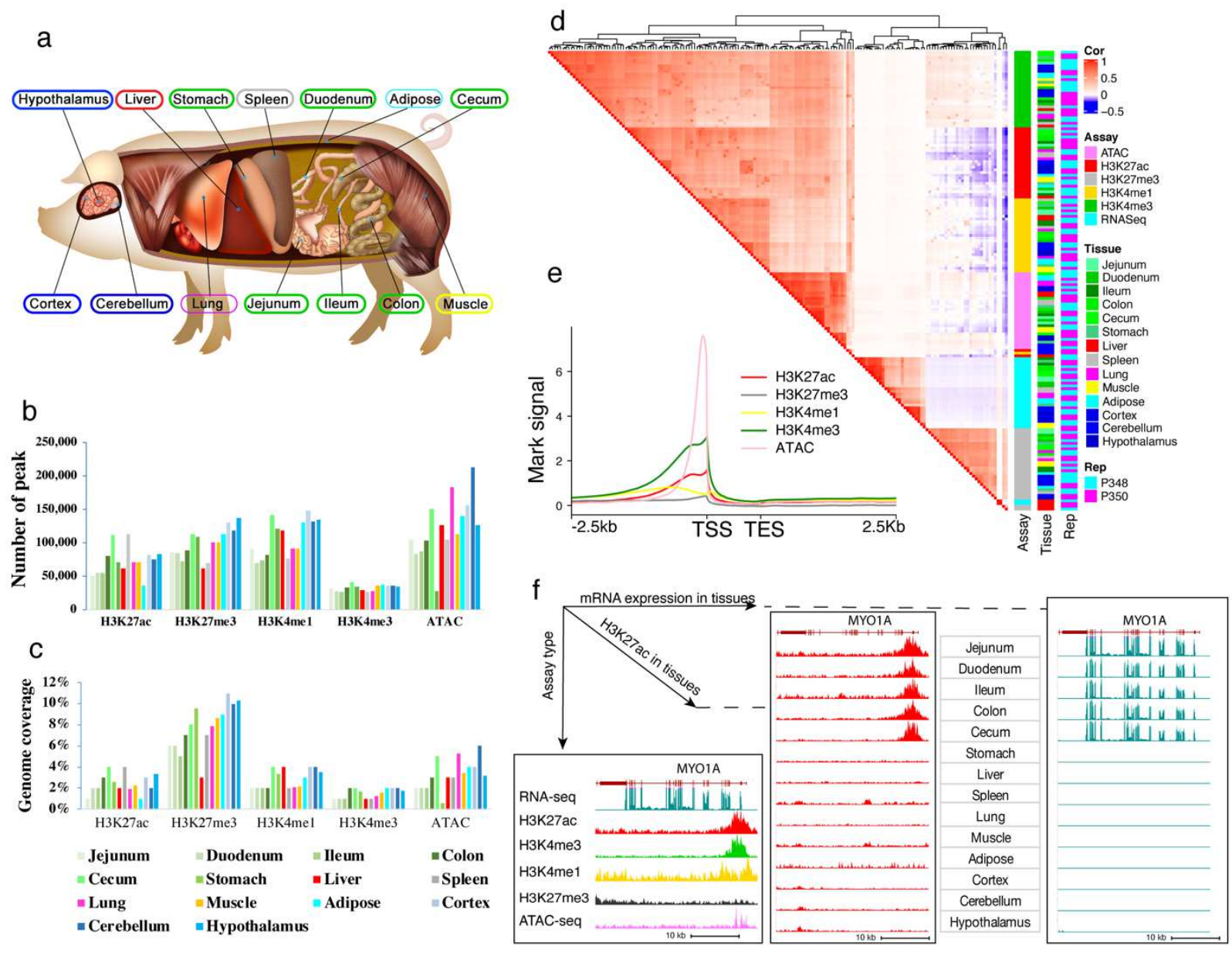

861

Fig.1 Data summary of epigenomic information across tissues and marks.

863 a, Tissues assayed by this study. b,c, Average peak number and genome coverage for each

864 epigenetic mark in each tissue. d, The Pearson correlations among assays, tissues, and biological

865 replicates (P348 and P350) based on the normalized signal in $1 \mathrm{~kb}$ windows stepped across the

866 whole genome. e, Average epigenetic mark signals proximal to protein coding genes. TSS,

867 transcription start site. TES, transcription end site. f, Epigenetic signal at the $M Y O 1 A$ locus

868 according to different assays and in different tissues. Vertical scale UCSC reads signal 0-200 for

869 RNA-seq, 0-100 for H3K27ac and H3K4me3, 0-50 for other marks and ATAC-seq. 

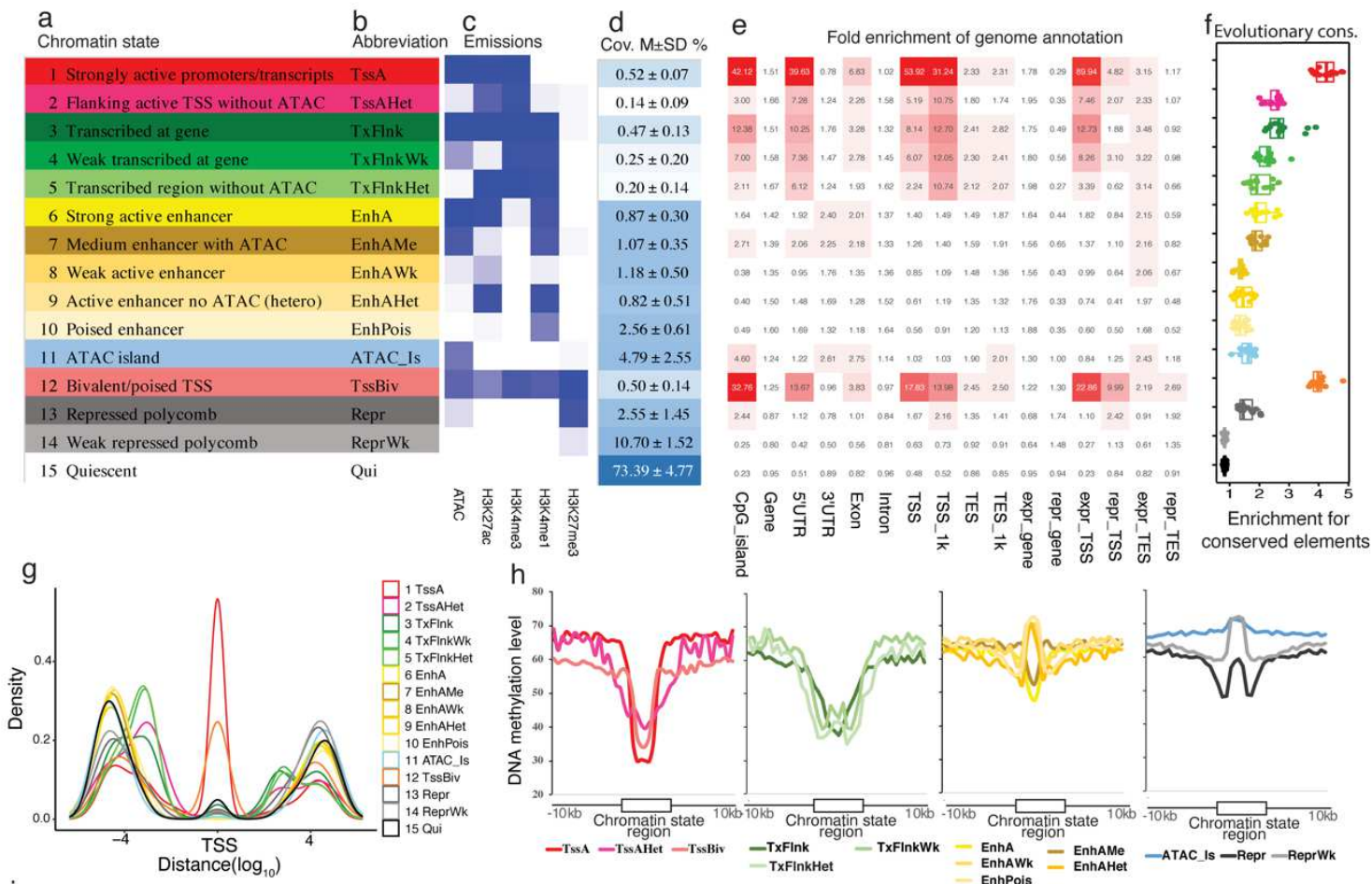

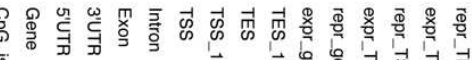

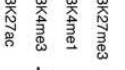
$\frac{\bar{w}}{\frac{2}{2}}$

i
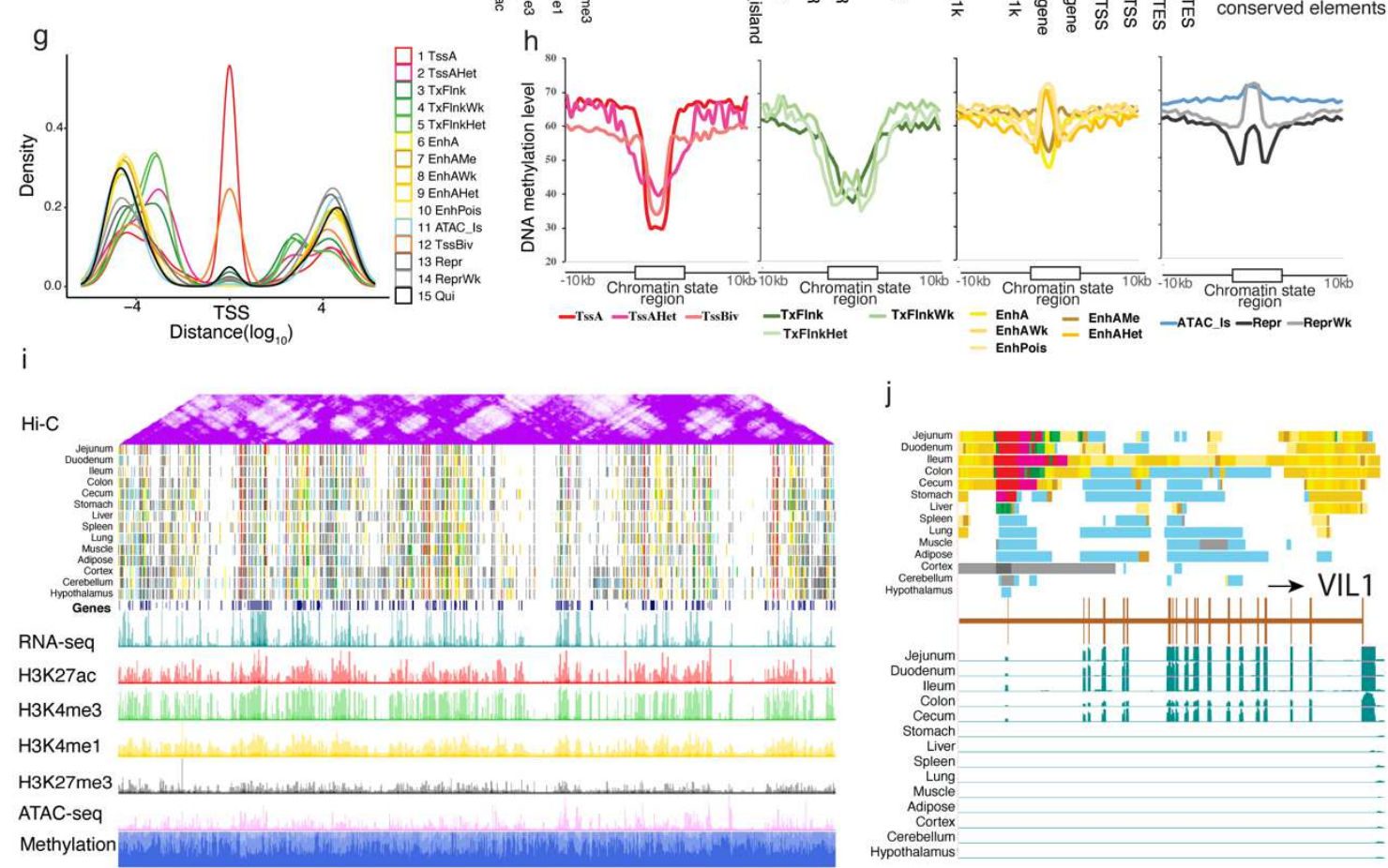

871 Fig.2 Chromatin landscape across 14 tissues.

872 a, b, Definitions and abbreviations of 15 chromatin states. c, Emission probabilities of individual

873 epigenetic marks for each chromatin state. The color from white to deep blue indicate emission

874 probability (0-1). d, Genomic coverages of each chromatin state. $\mathrm{M} \pm \mathrm{SD}$, means \pm standard

875 deviation e, Average enrichments of chromatin states for genomic annotations, including CpG

876 islands, genes, TSS/TES_1K $( \pm 1 \mathrm{~kb}$ around transcription start/end sites $)$, expressed genes

$877($ TPM $>=0.1)$, repressed genes $($ TPM $<0.1)$ in each tissue. f, Fold enrichments of chromatin states 
878 for non-coding mammalian conserved elements from Genomic Evolutionary Rate Profiling 879 (GERP). g, Density of each chromatin state in positions relative to gene transcription start sites 880 (TSS). h, Average methylation level of chromatin states in jejunum. i, Hi-C (250kb resolution), 881 predicted chromatin states, and epigenetic signal, and normalized methylation level landscape in 882 jejunum across the Chr7. j, Chromatin state landscapes and mRNA expression at VIL1 locus 883 (chr15:120,459,825-120,493,312, susScr11) across 14 tissues in pig. Vertical scale UCSC reads 884 signal 0-200 for RNA-seq.

885 886

887 888 

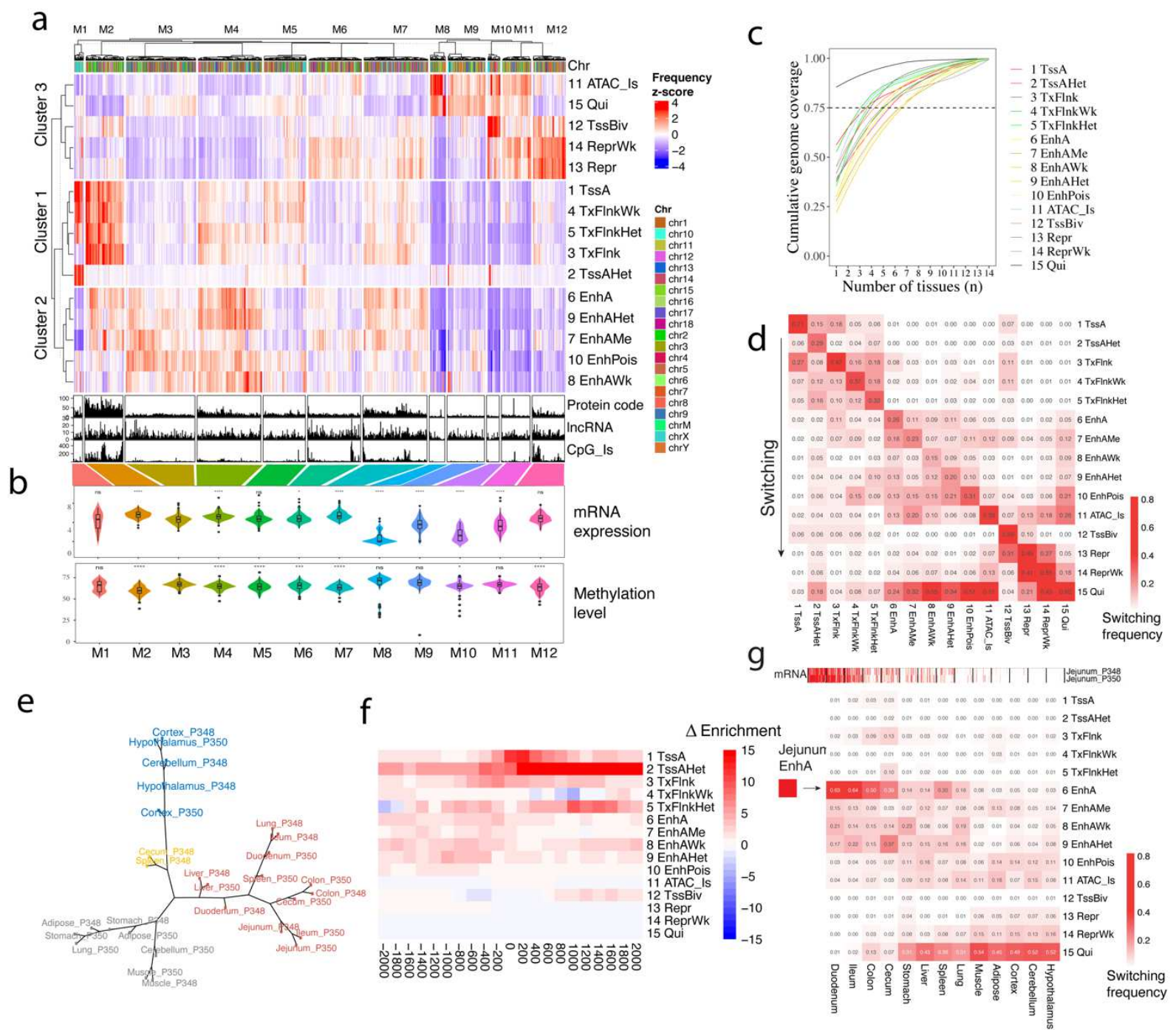

$890 \quad$ Fig.3 Genome-wide chromatin state dynamics across tissues.

891 a, Clustering of 2-Mb intervals (1,224 columns) into modules (M1-M12) based on average

892 chromatin state frequency across tissues in each interval. b, Average mRNA expression (TPM) of

893 genes and average methylation level of 2-Mb intervals belonging to each module. The statistical

894 significances for comparisons were calculated using t test, where “*” means $P<0.05$, “**” means

$895 P<0.01$, “***” means $P<0.001$. c, Chromatin state variability based on cumulative genome

896 coverage fraction. Dash line $=0.75$. d, Chromatin state switching between all tissues. e,

897 Hierarchical epigenome clustering using H3K4mel signal in EnhA states. f, Chromatin state 
898 enrichment relative to promoters of genes with jejunum-specific expression genes, relative to

899 muscle. g, Chromatin state switching of target enhancers (EnhA) of jejunum-specifically expressed

900 genes in other tissues.

901 


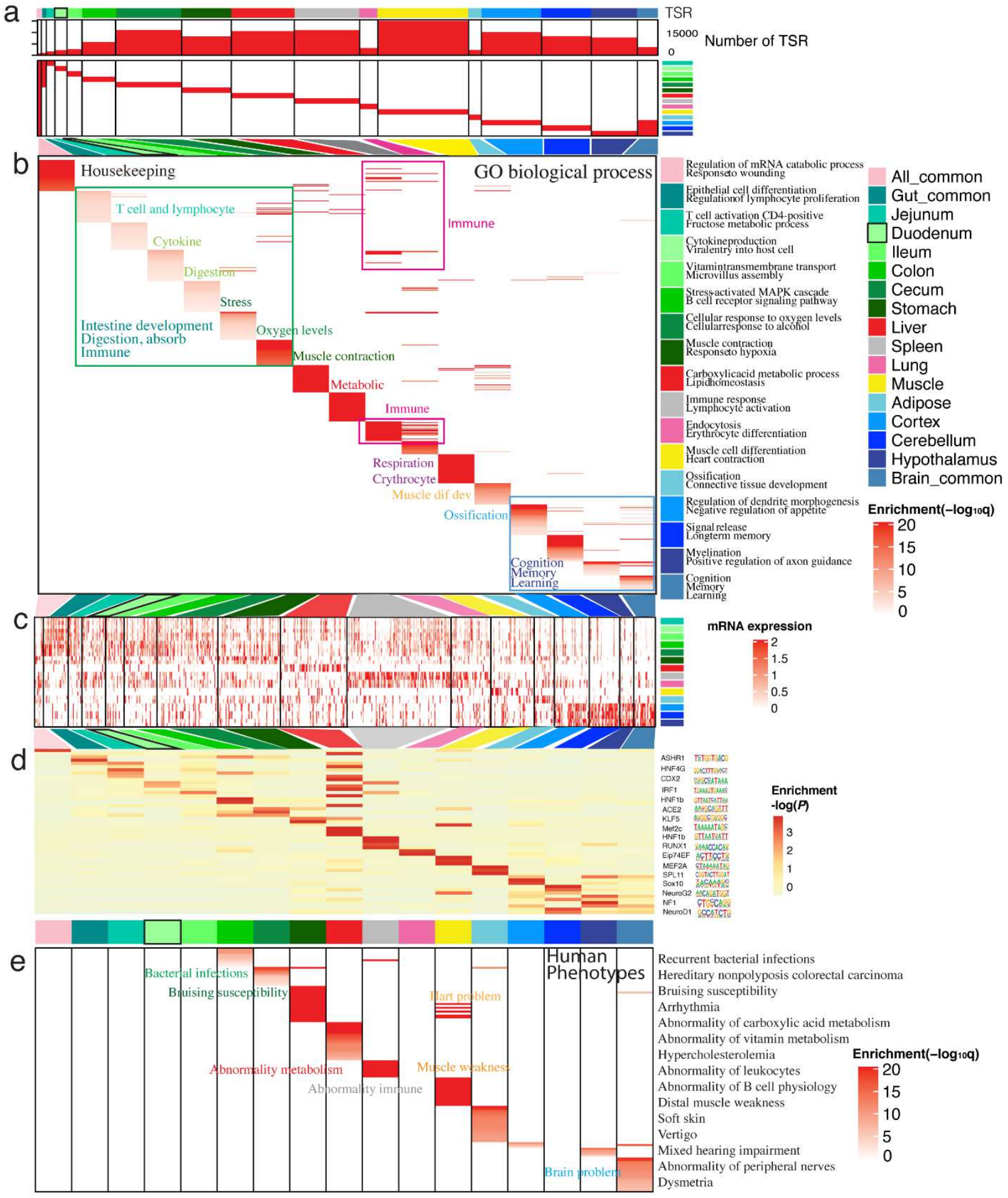


904 a, The number and enrichment distribution of 17 modules of TSR (strong enhancers (EnhA)) in

905 tissues. TSR: tissue-specific regulatory elements. The top colors represent 17 modules of strong

906 enhancers (column) referred to the legend on the right side. The side colors represent 14 tissues

907 (row) referred to the legend on the right side. b, Functional enrichment of proximal genes for each

908 module based on gene ontology (GO) biological processes. The columns represent 17 modules of

909 strong enhancers. The rows represent GO terms in each module. All GO terms are presented in

910 Supplementary table 5 (The notes in heatmap are summary function of nearby GO terms

911 enrichment (up-noted from jejunum to spleen, down-noted for lung, muscle and adipose)). c, The

912 mRNA expression (TPM) of EnhAs' putative target genes in each module. The columns represent

913 the genes in each module, the rows represent each tissue. d, The enrichment of transcription factor

914 motifs in each module. The columns represent 17 modules of EnhAs. The rows represent motif in

915 each module. All enriched motifs are presented in Supplementary Fig. 8a. e, Enrichment for human

916 phenotypes in each module, based on proximal genes. The columns represent 17 modules of

917 EnhAs. The rows represent phenotypes in each module. All phenotypes' enrichment are presented

918 in Supplementary table7. The notes in heatmap are summary function of nearby phenotypes

919 enrichment (each color stands for each tissue). 
a

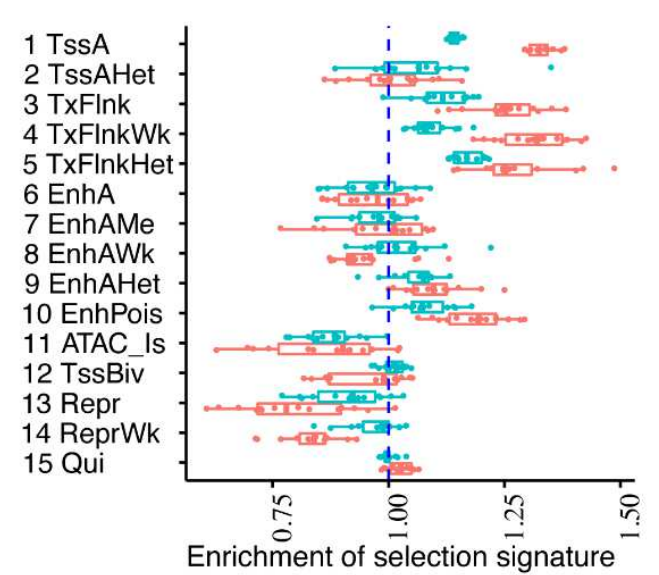

C

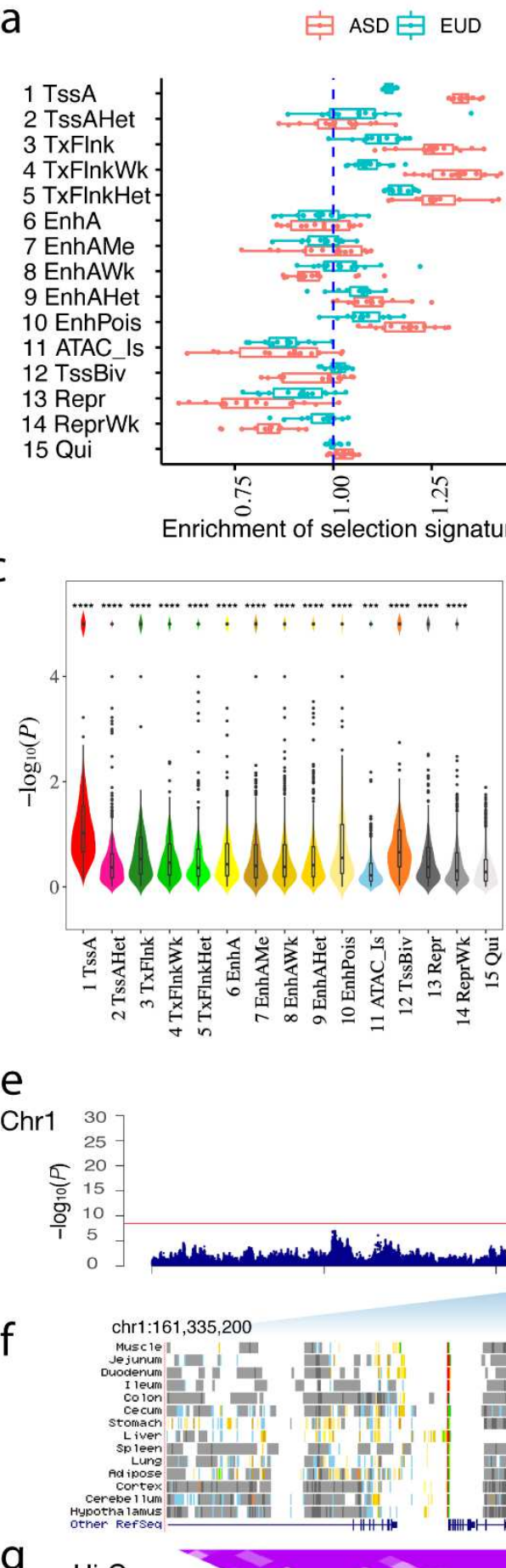

e

Chr1

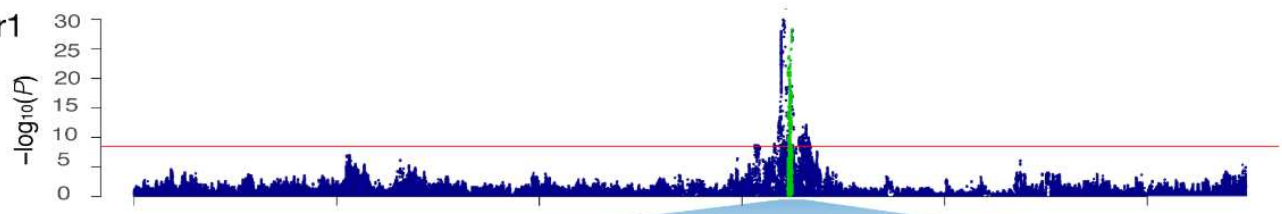

b
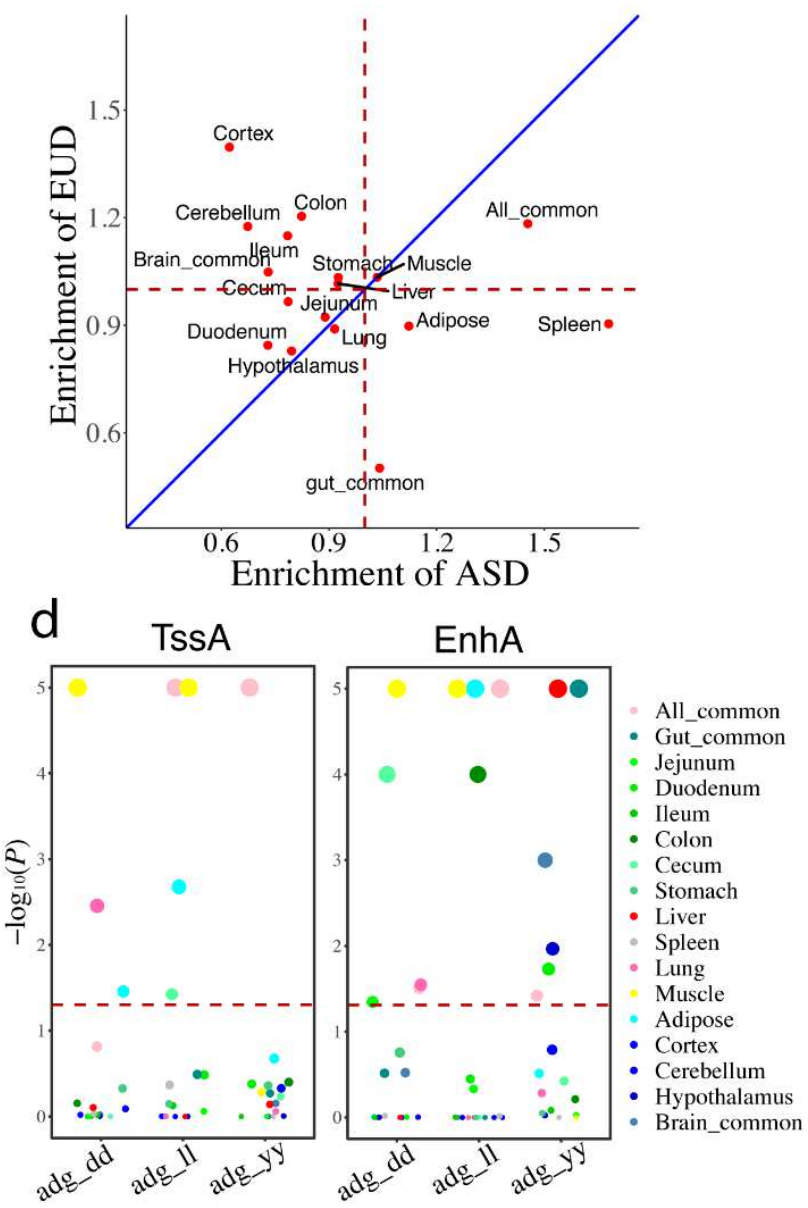

f

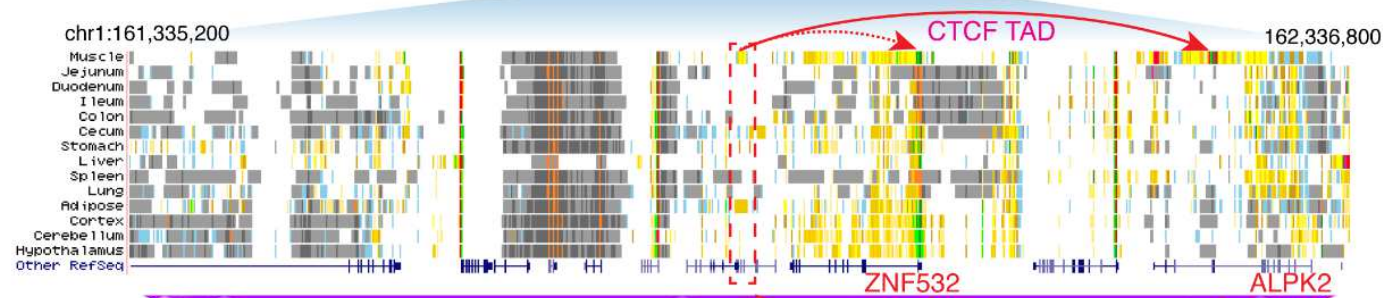

$9 \mathrm{Hi}-\mathrm{C}$

ALPK2

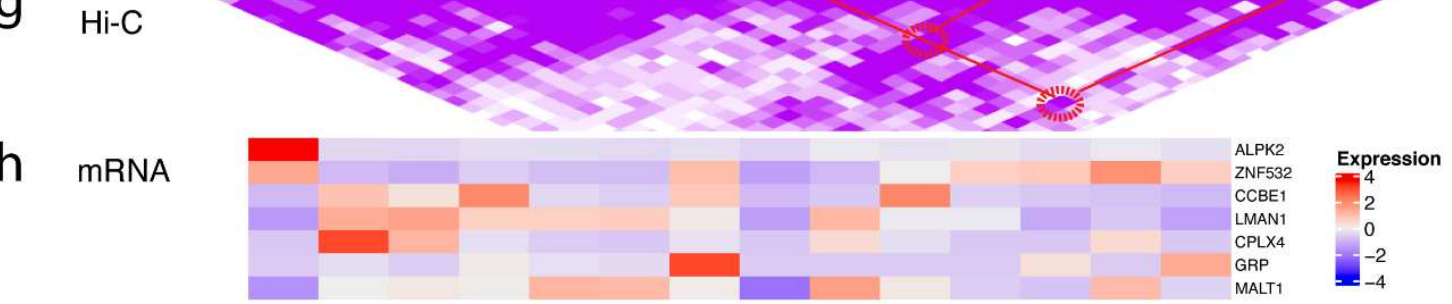

Muscle Jejunum Duodenum lleum Colon Cecum Stomach Liver Spleen Lung Adipose Cortex Cerebellum Hypothalamus 
921 Fig.5 Chromatin state plays an important role in pig domestication and complex traits.

922 a, Domestication selection signature enrichment of chromatin states in Asian and European pigs.

923 ASD: Asian pig domestication. EUD: European pig domestication. Dash-line = 1, above dash line

924 means significant enrichment. b, Domestication selection signature enrichment in tissue-specific

925 promoters (TssA) between Asian and European pigs. Dash-line $=1$, above 1 dash line means

926 significant enrichment. c, Genome-wide association studies (GWAS) signal enrichment within

927 chromatin states across 14 tissues and 44 complex traits in pigs. The statistical significances for

928 comparisons were calculated using a t test, where “***" means $P<0.001$. d, GWAS signal

929 enrichment of promoter (TssA) and strong enhancer (EnhA) tissue-specific regulatory elements

930 (TSR) in average daily gain (adg) of three pig populations (dd: Duroc, 1l: Landrace, yy: Yorkshire).

931 Dash line $=-\log _{10}(P=0.05)$, over dash line means significantly high enrichment. e, Manhattan plot

932 of average daily gain in the Landrace population. f, Chromatin states in the genomic region where

933 GWAS hits for each tissue (dashed rectangle box includes a muscle-specific enhance where SNPs

934 of GWAS hits locate; two arrows in red were predicted from CTCF TAD and H3K27ac signal that

935 suggest the muscle-specific enhancer may target ZNF532 and ALPK2). g. Hi-C loop (25kb

936 resolution) depiction between a muscle-specific enhancer and putative target genes. Purple shading

937 for the Hi-C data means loop intensity (auto-scale). Two highlighted Hi-C loop with the red circles

938 are potential contacts between a muscle-specific enhancer and ZNF532 and ALPK2. h, Expression

939 (normalized and centered TPM) of genes proximal to the muscle-specific enhancer. 
a

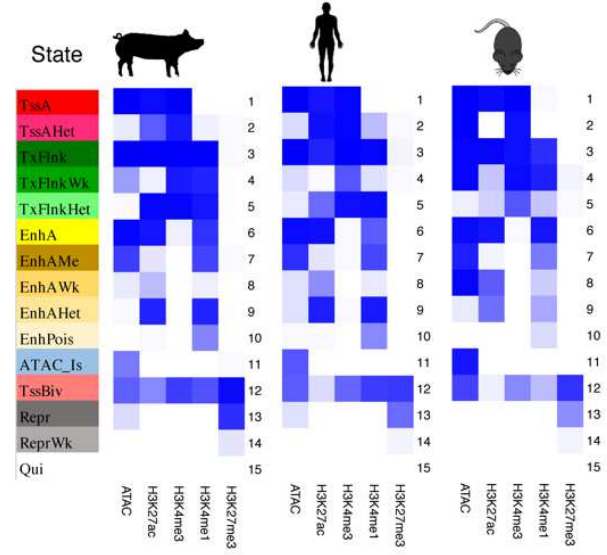

b

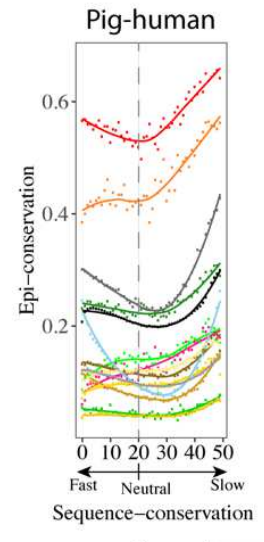

C

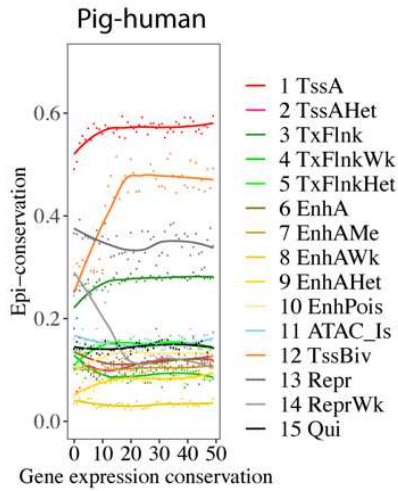

Gene expression conservatio d

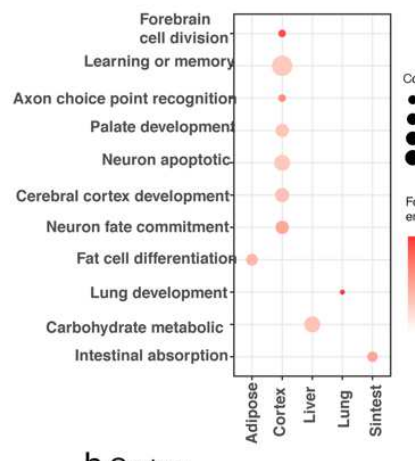

h Cortex

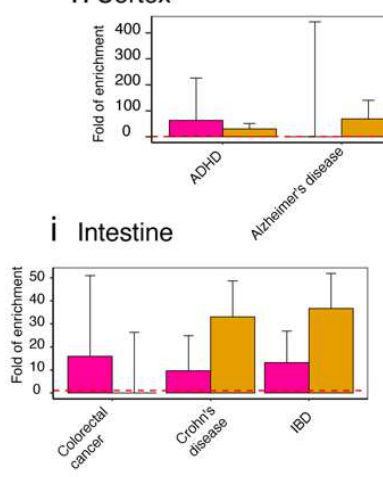

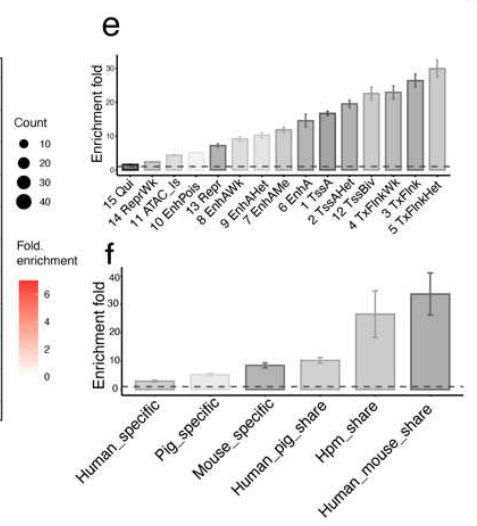

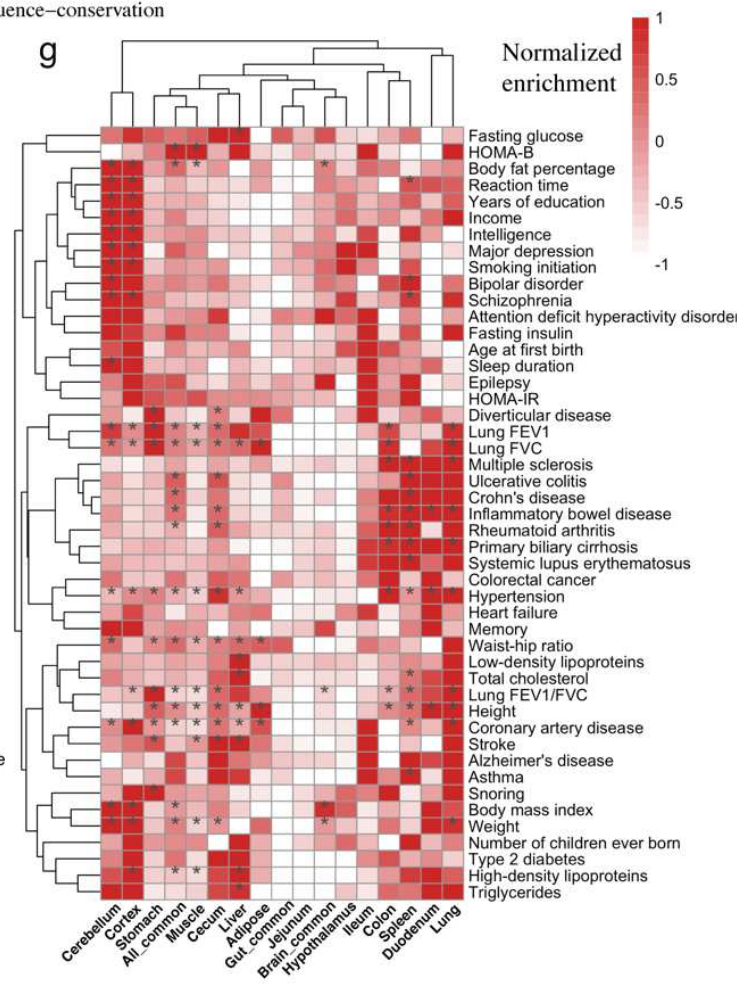

942 Fig.6 Interspecies conservation of chromatin states.

943 a, 15 chromatin states predicated in three species. The colors from white to deep blue indicate

944 emission probability from 0 to1. b, Relation between sequence conservation and epigenomic

945 conservation across six tissues. 50 genomic regions were ordered from the fastest changing $\left(0^{\text {th }}\right)$,

946 to neutral $\left(20^{\text {th }}\right)$, and to slowest changing $\left(49^{\text {th }}\right)$ in terms of sequence conservation

947 (Supplementary Fig. 13d). Epigenome conservation (see method) of chromatin states (b) within 
948 these regions was calculated for pig to human. Value in each region for each chromatin state was

949 then plotted. c, Relation between expression conservation and epigenomic conservation across

950 six tissues. Expression conservation was based on expression of 14,302 orthologous genes

951 among 3 species. Regions were ordered from the biggest difference in expression (0th), to the

952 smallest difference $\left(49^{\text {th }}\right)$. d, GO enrichment was based on genes proximal to $( \pm 2 \mathrm{~kb})$ human-

953 specific TssA in sequences of extreme conservation sets $\left(49^{\text {th }}\right)$. Count refers to the number of

954 genes. e, Human GWAS signal enrichment in different chromatin states. dash line=1, over dash

955 line means significant high enrichment. Error bars represent standard error around the estimates

956 of enrichment. Same meaning for dash line and error bars in following sub-figures. f, Human

957 GWAS enrichment in 6 groups of species-specific or shared EnhA. hpm_share stands for human-

958 pig-mouse shared. g, GWAS enrichment of pig tissue-specific enhancer (EnhA) in humans.

959 *means significant enrichment $(\mathrm{FDR}<0.05)$. h,i,j, Different GWAS enrichments between human-

960 pig and human-mouse shared strong enhancers (EnhA) in brain cortex, small intestine, and

961 adipose, respectively.

962

963 Supplementary information

964 Supplementary Information

965 Supplementary Figs. 1-16 and Tables 1-13

966

967 

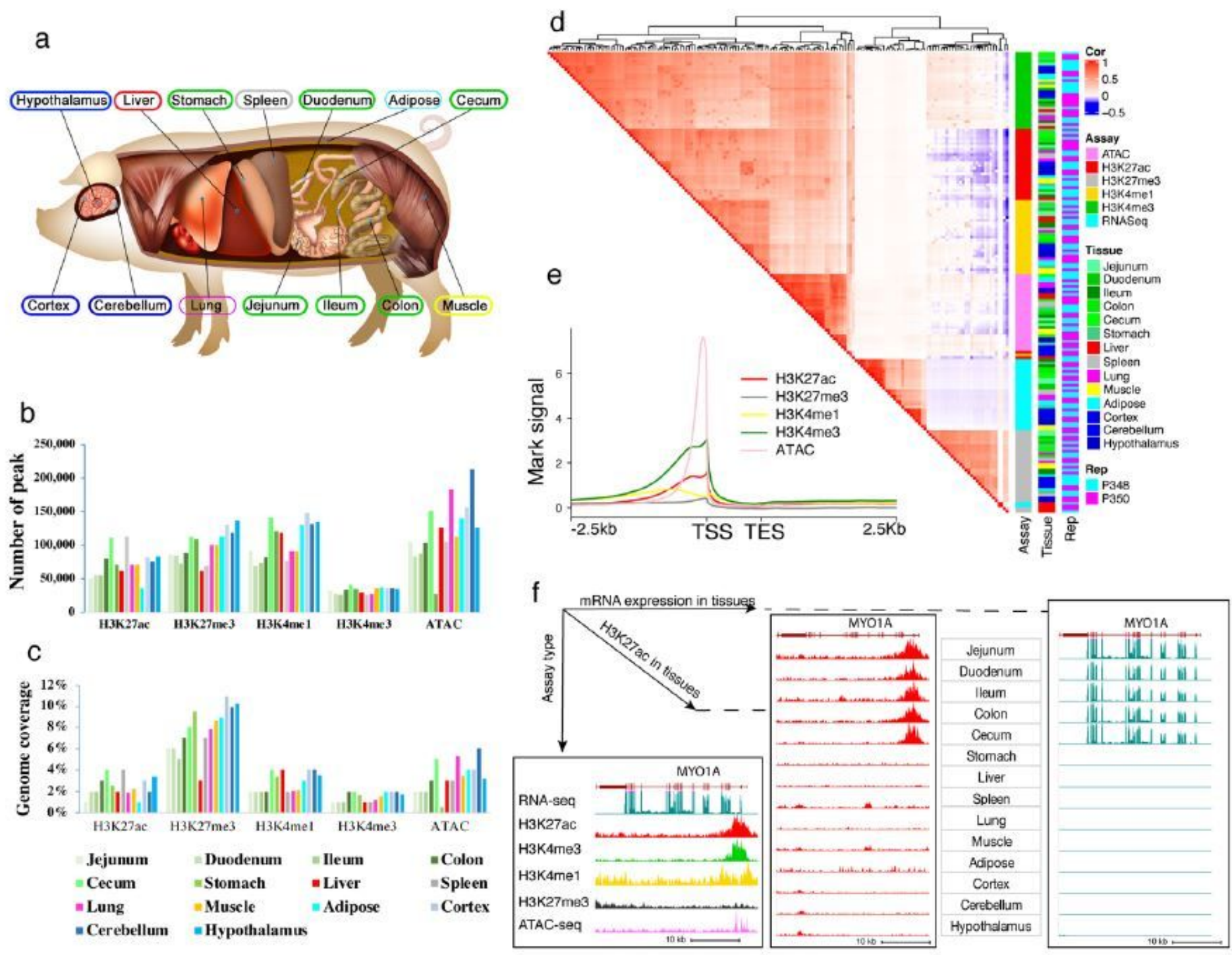

\section{Figure 1}

Fig.1 Data summary of epigenomic information across tissues and marks. a, Tissues assayed by this study. b,c, Average peak number and genome coverage for each epigenetic mark in each tissue. $d$, The Pearson correlations among assays, tissues, and biological replicates (P348 and P350) based on the normalized signal in $1 \mathrm{~kb}$ windows stepped across the whole genome. e, Average epigenetic mark signals proximal to protein coding genes. TSS, transcription start site. TES, transcription end site. f, Epigenetic signal at the MY01A locus according to different assays and in different tissues. Vertical scale UCSC reads signal 0-200 for RNA-seq, 0-100 for H3K27ac and H3K4me3, 0-50 for other marks and ATAC-seq. 

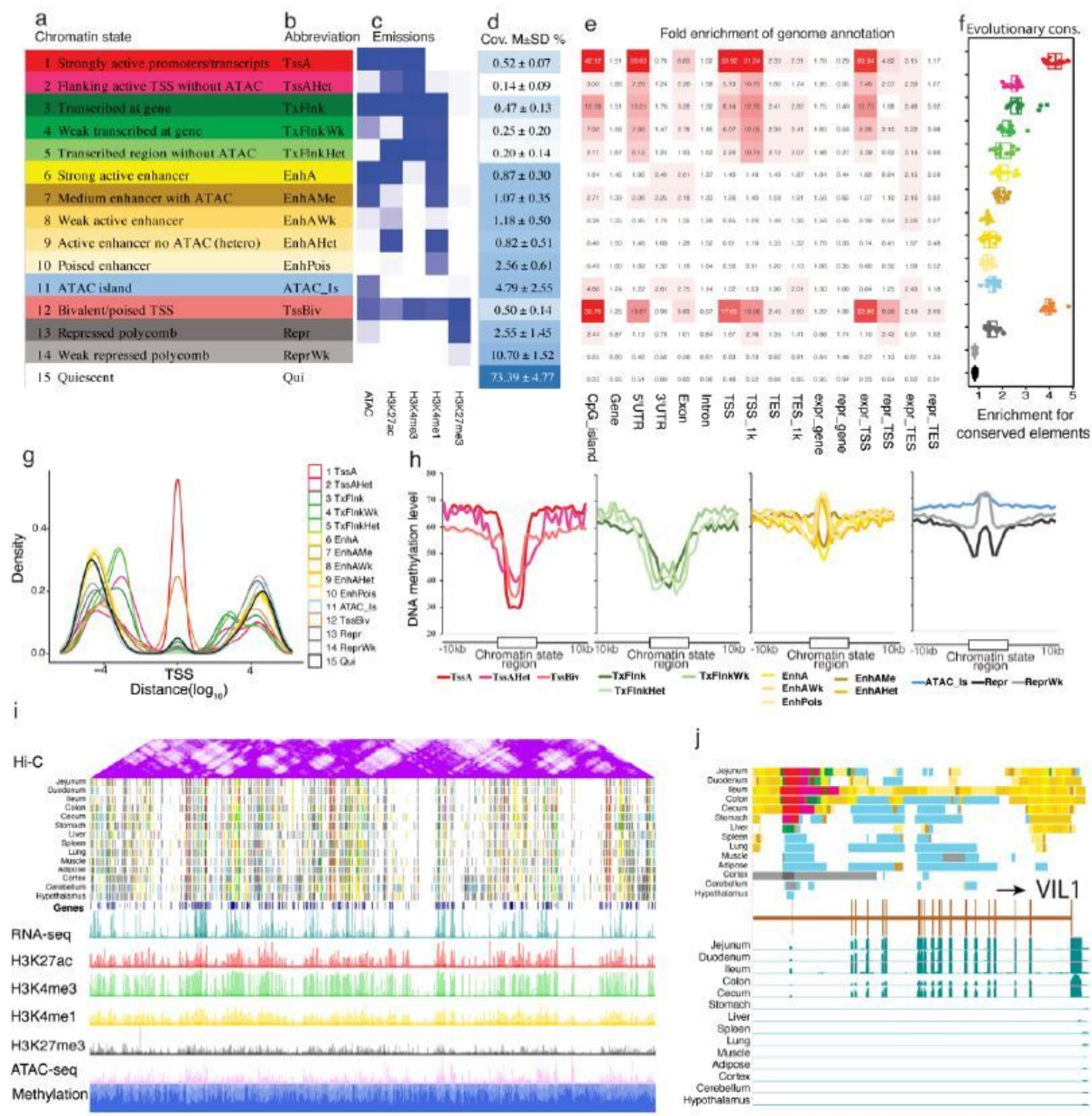

\section{Figure 2}

Chromatin landscape across 14 tissues. a, b, Definitions and abbreviations of 15 chromatin states. c, Emission probabilities of individual epigenetic marks for each chromatin state. The color from white to deep blue indicate emission probability (0-1). d, Genomic coverages of each chromatin state. $M \pm S D$, means \pm standard deviation e, Average enrichments of chromatin states for genomic annotations, including $\mathrm{CpG}$ islands, genes, TSS/TES_1K ( $\pm 1 \mathrm{~kb}$ around transcription start/end sites), expressed genes (TPM >=0.1), repressed genes (TPM < 0.1) in each tissue. f, Fold enrichments of chromatin states 42 for non-coding mammalian conserved elements from Genomic Evolutionary Rate Profiling (GERP). g, Density of each chromatin state in positions relative to gene transcription start sites (TSS). h, Average 
methylation level of chromatin states in jejunum. i, Hi-C (250kb resolution), predicted chromatin states, and epigenetic signal, and normalized methylation level landscape in jejunum across the Chr7. j, Chromatin state landscapes and mRNA expression at VIL1 locus (chr15:120,459,825-120,493,312, susScr11) across 14 tissues in pig. Vertical scale UCSC reads signal 0-200 for RNA-seq.

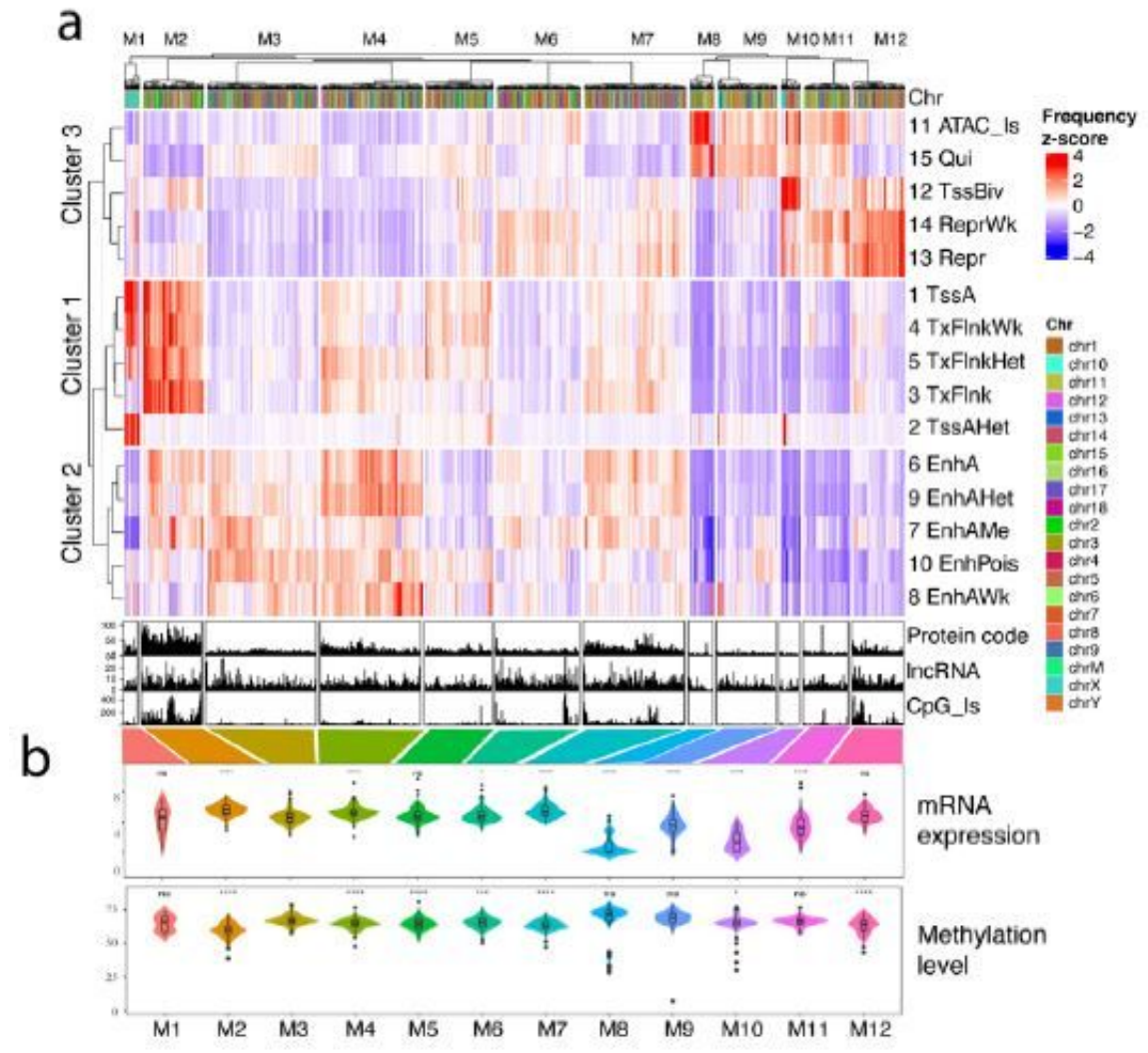

C
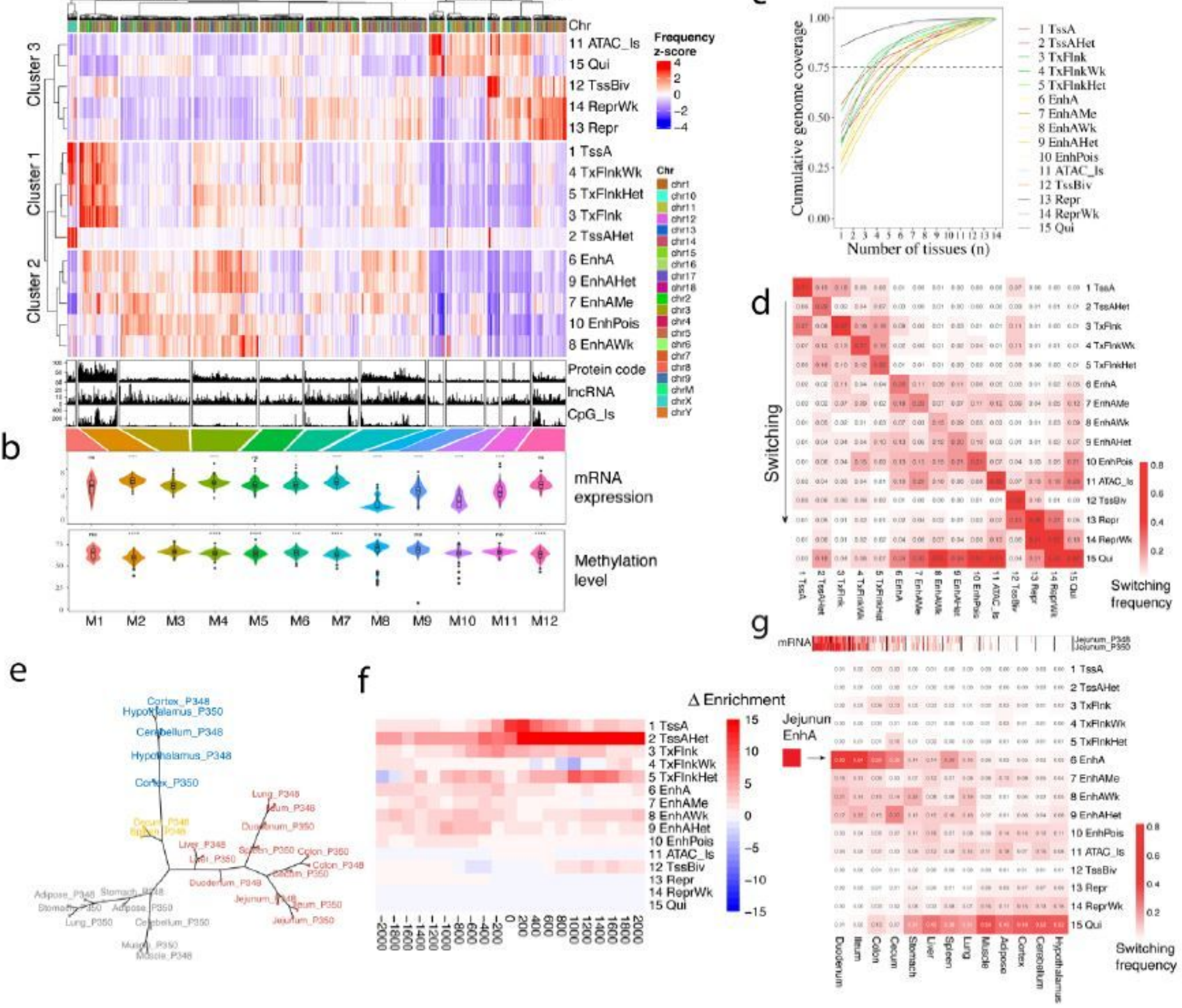

Figure 3

Genome-wide chromatin state dynamics across tissues. a, Clustering of 2-Mb intervals (1,224 columns) into modules (M1-M12) based on average chromatin state frequency across tissues in each interval. b, Average mRNA expression (TPM) of genes and average methylation level of 2-Mb intervals belonging to each module. The statistical significances for comparisons were calculated using $t$ test, where " $\star$ " means

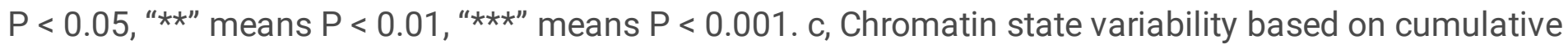
genome coverage fraction. Dash line $=0.75$. $d$, Chromatin state switching between all tissues. $e$, 
Hierarchical epigenome clustering using H3K4me1 signal in EnhA states. f, Chromatin state 44 enrichment relative to promoters of genes with jejunum-specific expression genes, relative to muscle. $g$, Chromatin state switching of target enhancers (EnhA) of jejunum-specifically expressed genes in other tissues.

a

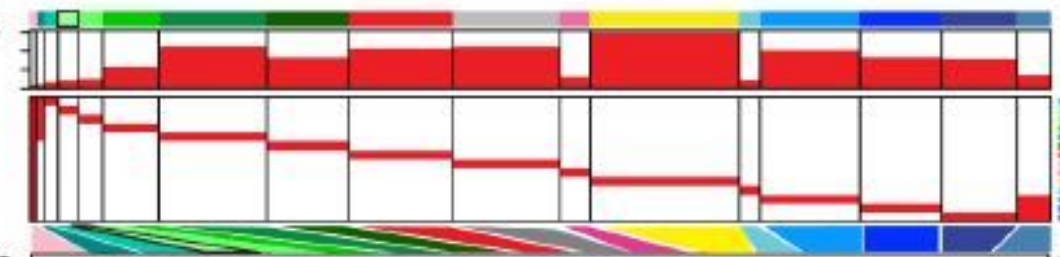

b
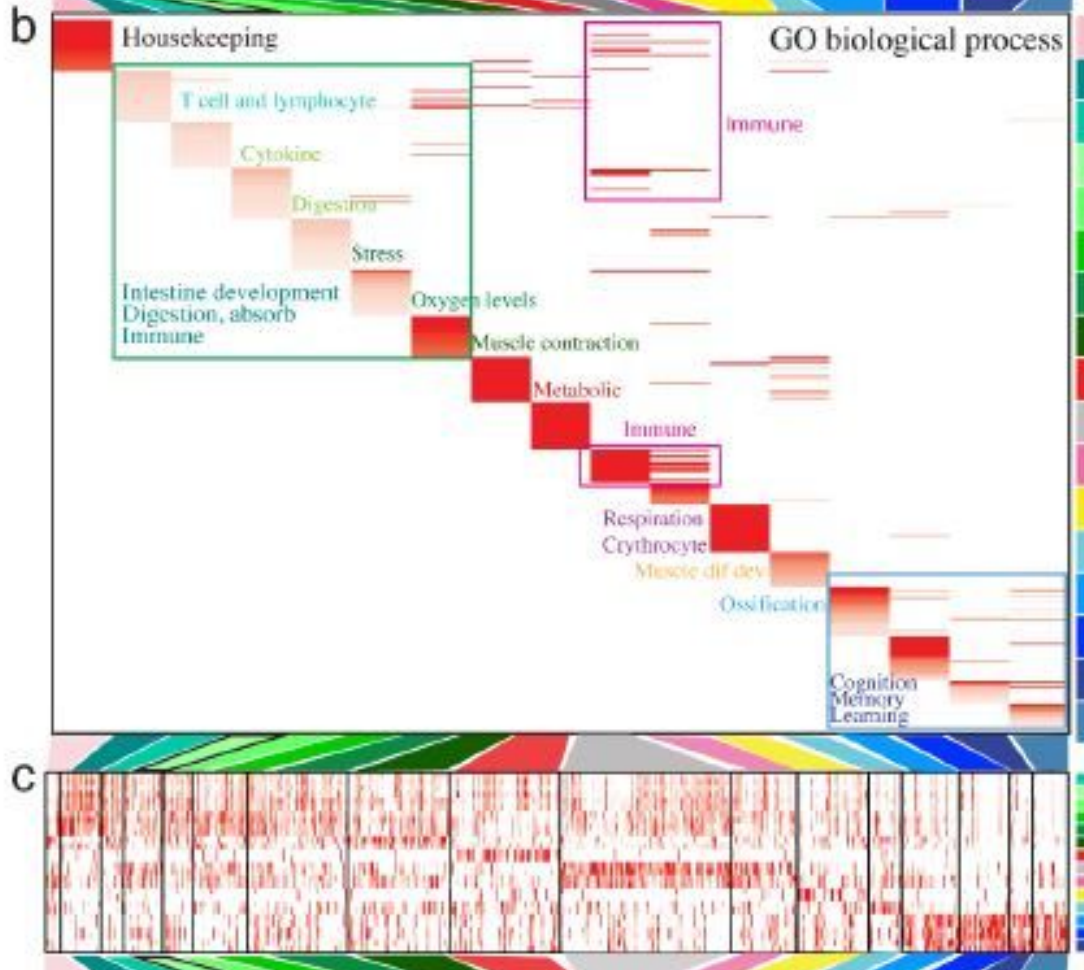

18000 Number of TSP
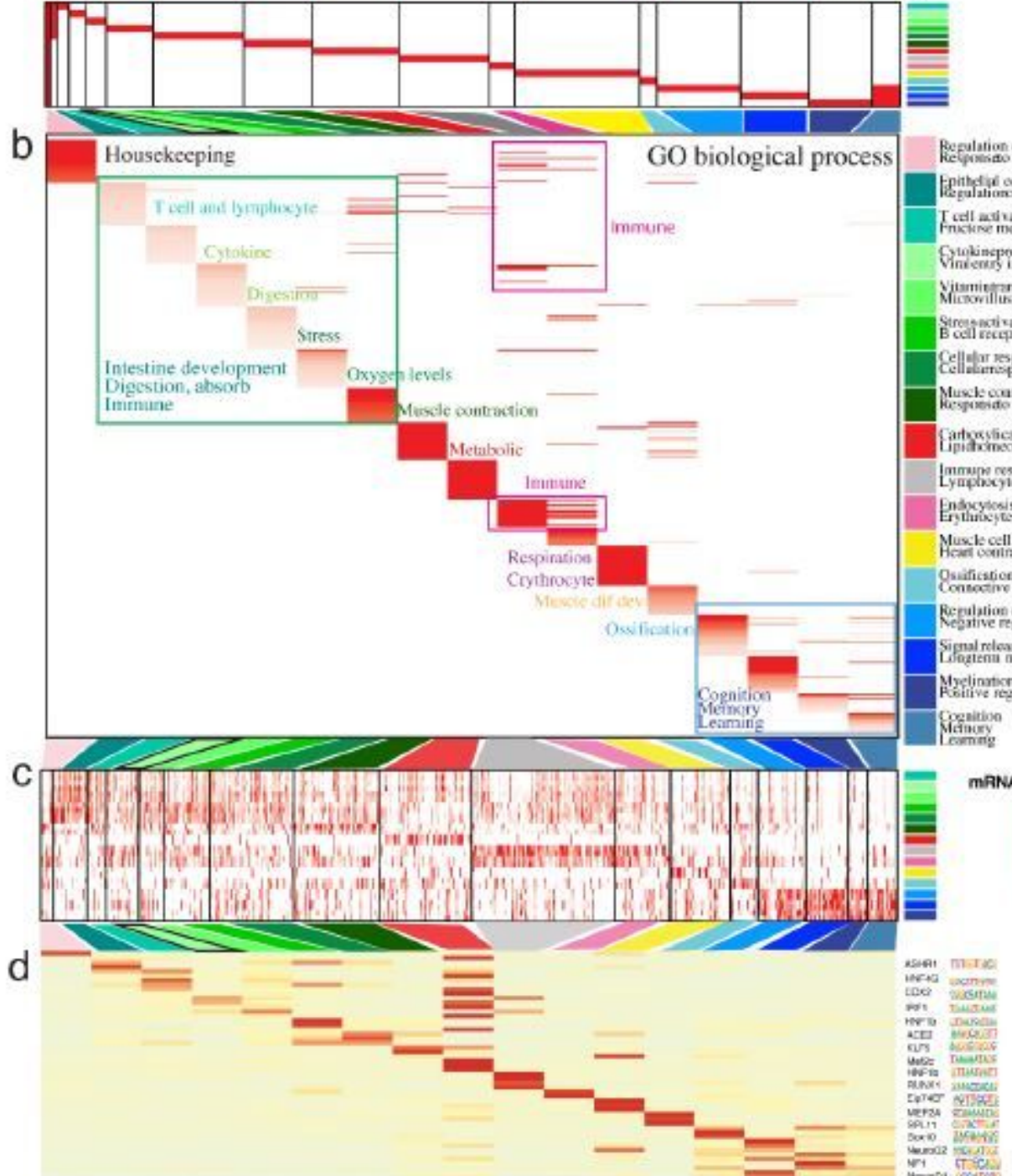

Pesulation of miRNA envabolic proses

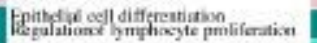

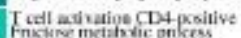

Gimkincroduction

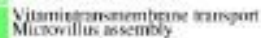

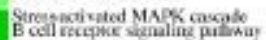

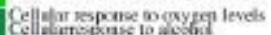

Muscle onanaciog

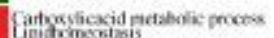

Lymupresponom

Enbogsonis diferentiation

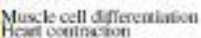

Conitication

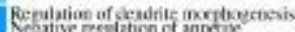

All_common

Gut_common

Jejunum

Duodenum

lleum

Colon

Cecum

Stomach

Liver

Spleen

Lung

Muscle

Adipose

Cortex

Cerebellum

Hypothalamus

Brain_common

Eirgul relevese

Myelinticn

$-20$

Cuandivon

mANA expression

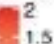

1.5

1

0.5
0

e
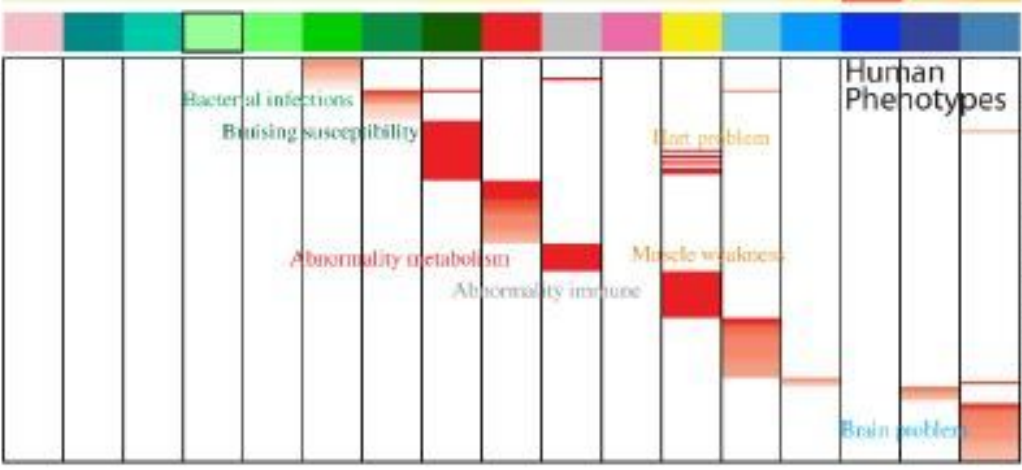

Recurvent tacterial infections

Enrichment

$-\log (P)$

3

Hereditary tonpolyposis colonetal earcinome

Bruising susccotibility

Arrhythmia

A beormality of carboxylic axid metsoolism

Abeormulity of vitamin metsobolism

Hypercholesierolemia Enrichment( $-\log n q)$

Aboxirmality of leakncytes

Abocormality of B cell physiology

Distal muscle weakness

Soft skin

verion

Mixed learing impoirmen

A boxmmality of periphenil nerves

20

15

10

5

Figure 4 
Tissue-specific strong enhancers (EnhA) and their potential functions in 14 tissues. 46 a, The number and enrichment distribution of 17 modules of TSR (strong enhancers (EnhA)) in tissues. TSR: tissue-specific regulatory elements. The top colors represent 17 modules of strong enhancers (column) referred to the legend on the right side. The side colors represent 14 tissues (row) referred to the legend on the right side. b. Functional enrichment of proximal genes for each module based on gene ontology (GO) biological processes. The columns represent 17 modules of strong enhancers. The rows represent $\mathrm{GO}$ terms in each module. All GO terms are presented in Supplementary table 5 (The notes in heatmap are summary function of nearby GO terms enrichment (up-noted from jejunum to spleen, down-noted for lung, muscle and adipose)). c, The mRNA expression (TPM) of EnhAs' putative target genes in each module. The columns represent the genes in each module, the rows represent each tissue. $d$, The enrichment of transcription factor motifs in each module. The columns represent 17 modules of EnhAs. The rows represent motif in each module. All enriched motifs are presented in Supplementary Fig. 8a. e, Enrichment for human phenotypes in each module, based on proximal genes. The columns represent 17 modules of EnhAs. The rows represent phenotypes in each module. All phenotypes' enrichment are presented in Supplementary table7. The notes in heatmap are summary function of nearby phenotypes enrichment (each color stands for each tissue). 
a

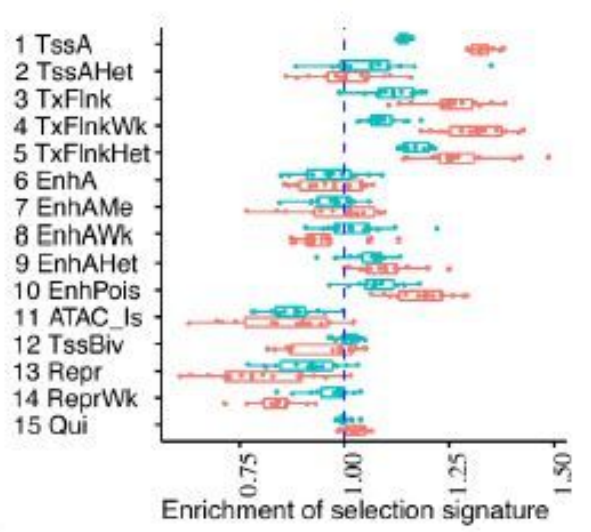

C

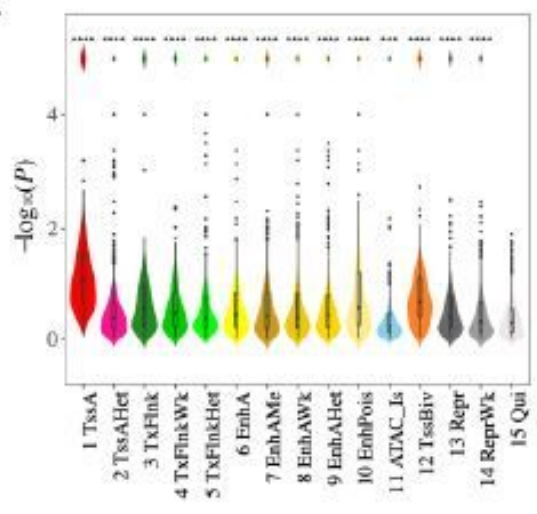

b
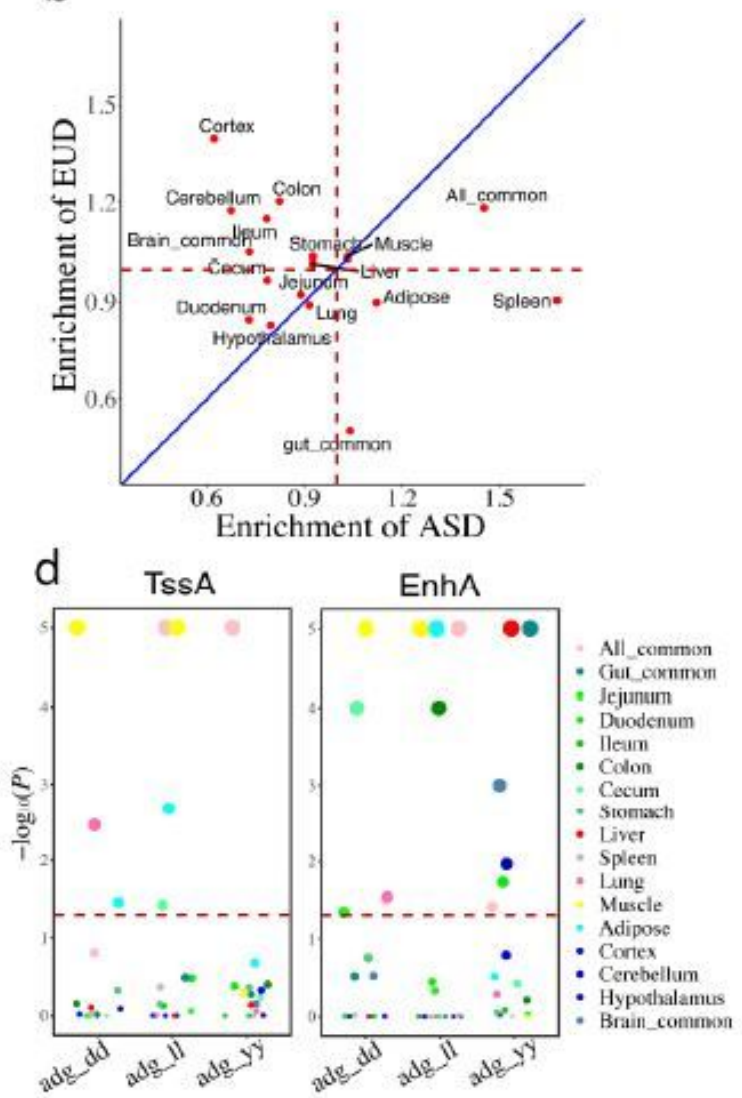

e

Chr1
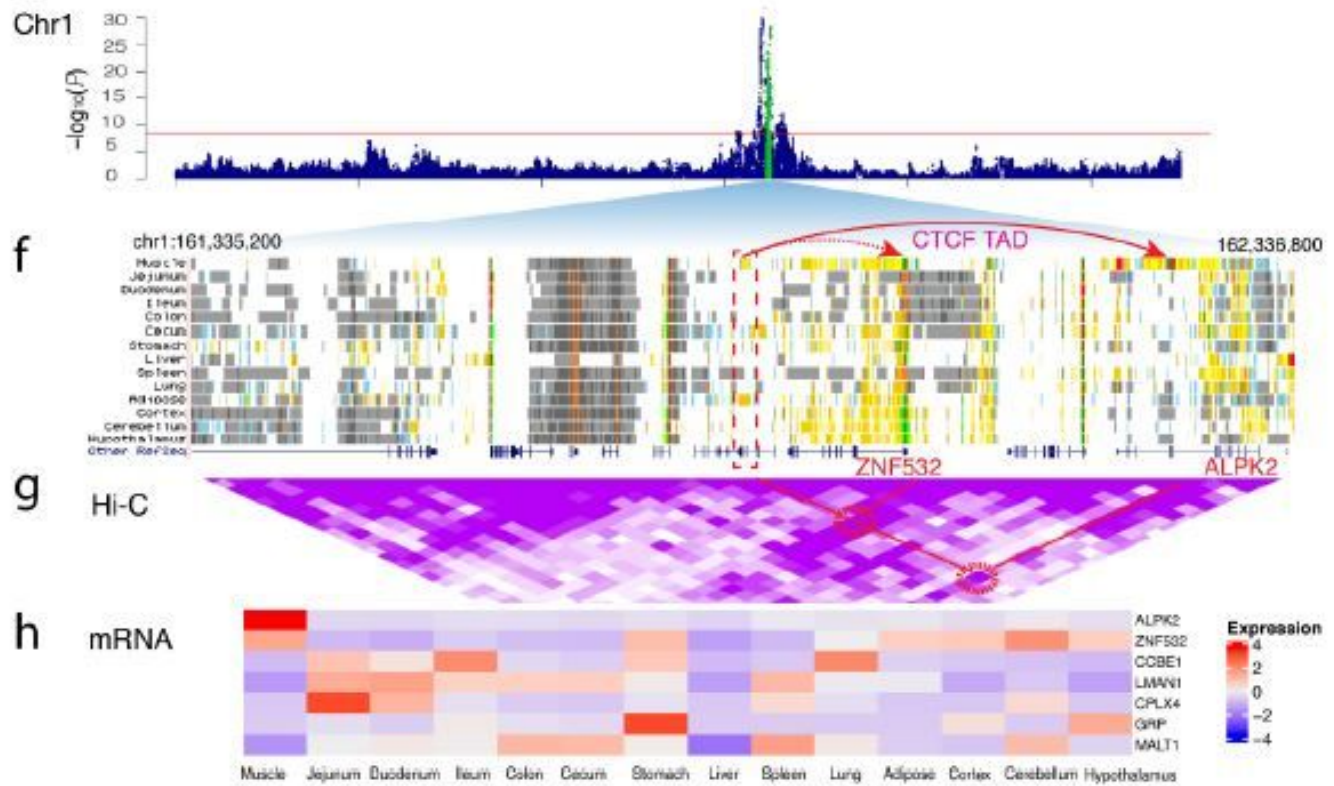

Figure 5

Chromatin state plays an important role in pig domestication and complex traits. a, Domestication selection signature enrichment of chromatin states in Asian and European pigs. ASD: Asian pig domestication. EUD: European pig domestication. Dash-line = 1, above dash line means significant enrichment. b, Domestication selection signature enrichment in tissue-specific promoters (TssA) between Asian and European pigs. Dash-line $=1$, above 1 dash line means significant enrichment. c, Genome-wide 
association studies (GWAS) signal enrichment within chromatin states across 14 tissues and 44 complex traits in pigs. The statistical significances for comparisons were calculated using a t test, where " $\star \star \star$ " means $P<0.001$. $d$, GWAS signal enrichment of promoter (TssA) and strong enhancer (EnhA) tissuespecific regulatory elements (TSR) in average daily gain (adg) of three pig populations (dd: Duroc, II: Landrace, yy: Yorkshire). Dash line=-log10 $(P=0.05)$, over dash line means significantly high enrichment. e, Manhattan plot of average daily gain in the Landrace population. $\mathrm{f}$, Chromatin states in the genomic region where GWAS hits for each tissue (dashed rectangle box includes a muscle-specific enhance where SNPs of GWAS hits locate; two arrows in red were predicted from CTCF TAD and H3K27ac signal that suggest the muscle-specific enhancer may target ZNF532 and ALPK2). g. Hi-C loop (25kb resolution) depiction between a muscle-specific enhancer and putative target genes. Purple shading for the Hi-C data means loop intensity (auto-scale). Two highlighted Hi-C loop with the red circles are potential contacts between a muscle-specific enhancer and ZNF532 and ALPK2. h, Expression (normalized and centered TPM) of genes proximal to the muscle-specific enhancer. 
a

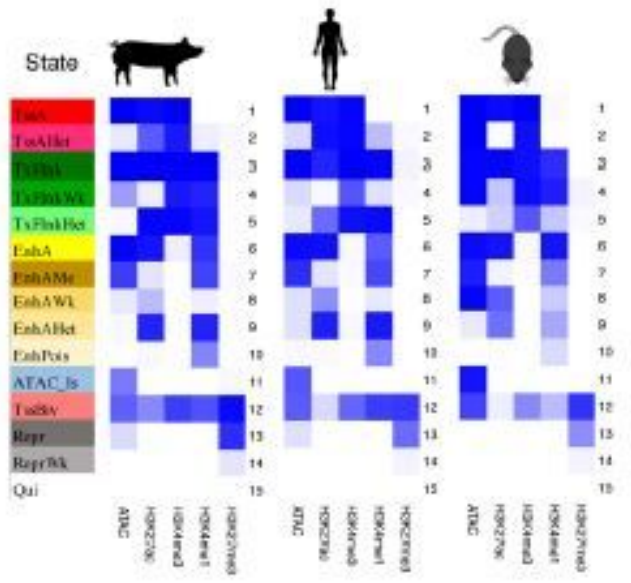

b

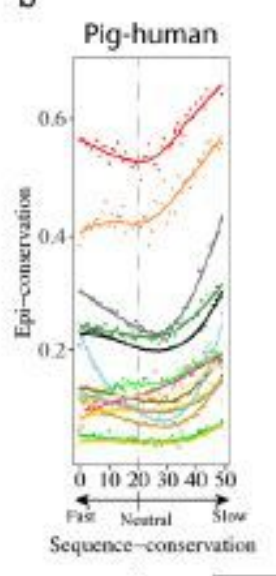

C

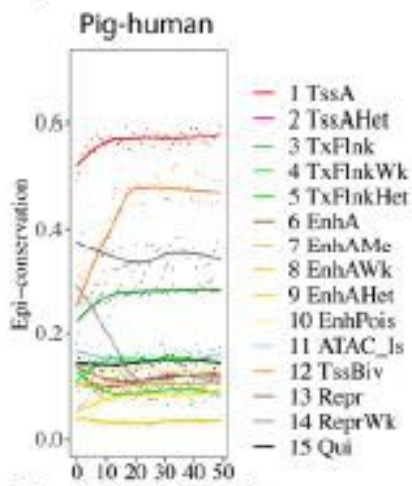

Gene expression conservation

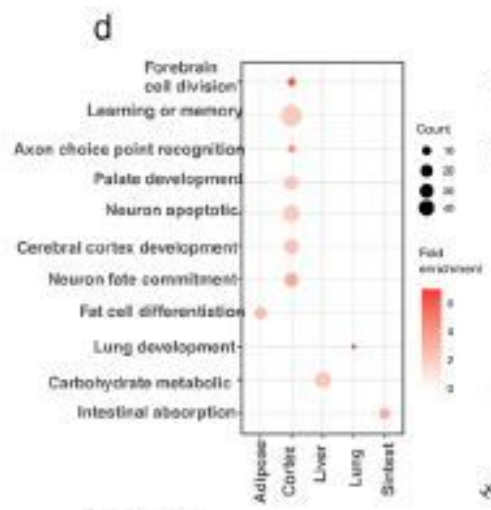

h Cortex

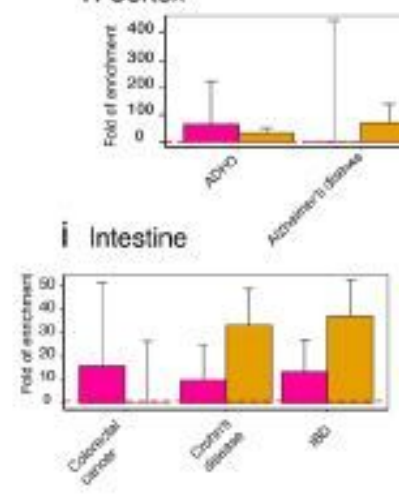

e

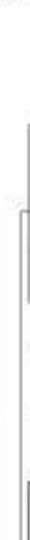

g

(2)

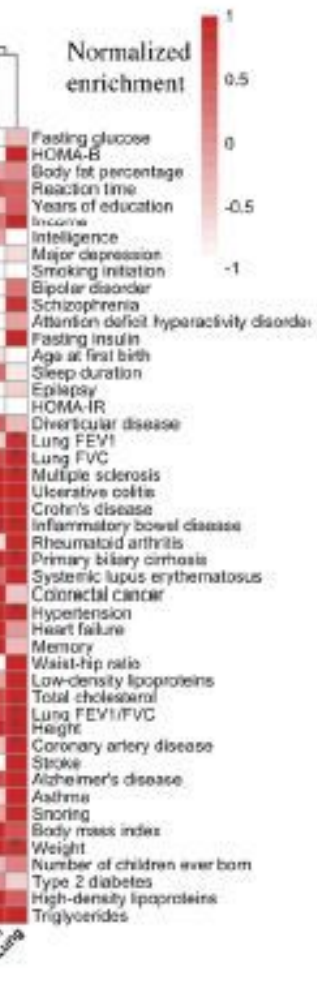

\section{Figure 6}

Interspecies conservation of chromatin states. a, 15 chromatin states predicated in three species. The colors from white to deep blue indicate emission probability from 0 to 1 . b, Relation between sequence conservation and epigenomic conservation across six tissues. 50 genomic regions were ordered from the fastest changing (0th), to neutral (20th), and to slowest changing (49th) in terms of sequence conservation (Supplementary Fig. 13d). Epigenome conservation (see method) of chromatin states (b) within 50 these regions was calculated for pig to human. Value in each region for each chromatin state was then plotted. c, Relation between expression conservation and epigenomic conservation across six tissues. Expression conservation was based on expression of 14,302 orthologous genes among 3 species. Regions were ordered from the biggest difference in expression (0th), to the smallest difference (49th). d, GO enrichment was based on genes proximal to ( $\pm 2 \mathrm{~kb}$ ) human specific TssA in sequences of 
extreme conservation sets (49th). Count refers to the number of genes. e, Human GWAS signal enrichment in different chromatin states. dash line $=1$, over dash line means significant high enrichment. Error bars represent standard error around the estimates of enrichment. Same meaning for dash line and error bars in following sub-figures. f, Human GWAS enrichment in 6 groups of species-specific or shared EnhA. hpm_share stands for human958 pig-mouse shared. g, GWAS enrichment of pig tissue-specific enhancer $(E n h A)$ in humans. *means significant enrichment (FDR<0.05). h,i,j, Different GWAS enrichments between human pig and human-mouse shared strong enhancers (EnhA) in brain cortex, small intestine, and adipose, respectively.

\section{Supplementary Files}

This is a list of supplementary files associated with this preprint. Click to download.

- 3SupplementaryFig.pdf

- 4SupplementaryTable.xlsx 